\title{
Numerical and Analytic Method for Solving Proposal Fuzzy Nonlinear Volterra Integral Equation by Using Homotopy Analysis Method
}

\author{
Sameer Qasim Hasan, Alan Jalal Abdulqader \\ College of Education, Department of Mathematics, Al-Mustansiriya University, Baghdad, Iraq \\ Email address: \\ dr.sameer_Kkasim@yahoo.com (S. Q. Hasan), alanjala1515@yahoo.com (A. J. Abdulqader)
}

\section{To cite this article:}

Sameer Qasim Hasan, Alan Jalal Abdulqader. Numerical and Analytic Method for Solving Proposal Fuzzy Nonlinear Volterra Integral Equation by Using Homotopy Analysis Method. American Journal of Applied Mathematics. Vol. 4, No. 3, 2016, pp. 142-157. doi: 10.11648/j.ajam.20160403.15

Received: May 4, 2016; Accepted: May 14, 2016; Published: May 28, 2016

\begin{abstract}
In this paper, we presented the convergence of the solution for the nonlinear fuzzy volterra integral equation with high computational and complexity to find the solution in analytical method, so we describable this solution by using Homotopy analysis method, by using Banach fixed point theory for existence and uniqueness. That with explained numerical examples. Finally using the MAPLE program to solve our problem.
\end{abstract}

Keywords: Fuzzy Number, Volterra nonlinear Integral Equation, Operator of Fuzzy Number, Fuzzy Integral, Homotopy Analysis Method

\section{Introduction}

The fuzzy integral equations are one of the important part of the fuzzy analysis theory that play major role in numerical analysis. The concept of fuzzy numbers and arithmetic operations on it was introduced by Zadeh [1] which was further enriched by Mizumoto and Tanaka [24]. Dubois and Prade [21] made a significant contribution by introducing the concept of LR fuzzy numbers and presented a computational formula for operations on fuzzy numbers. Also they $[4,7,8]$ was introduced the concept of integration of fuzzy functions. Later, Goetschel and Voxman [5] preferred a Riemann integral type approach, Kaleva [25] chose to define the integral of fuzzy function, using the Lebesgue-type concept for integration. One of the first applications of fuzzy integration was given by $\mathrm{Wu}$ and $\mathrm{Ma}$ who investigated the fuzzy Fredholm integral equation of the second kind. Recently, some mathematician have studied solution of fuzzy integral equation by numerical method $[1,9,13]$. In present, we try to employ homotopy analysis method for solving new formula fuzzy nonlinear Volterra integral equation. Furthermore, also we study the existence of a unique solution and convergence of the HAM methods for new formula fuzzy nonlinear Volterra integral equation.

\section{Basic Concepts}

Basic definitions of fuzzy number are given in $[1,2,10$, $15,17,20]$ as follows:

Definition 2.1. Fuzzy number. A fuzzy number is a map $u: R \rightarrow[a, b]$, which satisfying

(1) $\mathrm{u}$ is upper semi- continuous function,

(2) $\mathrm{u}(\mathrm{x})=0$ outside some interval $[a, d]$

(3) There are real numbers b, c such $a \leq b \leq c \leq d$

i) $\mathrm{u}(\mathrm{x})$ is a monotonic increasing function on $[a, b]$

ii) $\mathrm{u}(\mathrm{x})$ is a monotonic decreasing function on $[c, d]$

iii) $\mathrm{u}(\mathrm{x})=1$ for all $x \in[b, c]$

The set of all fuzzy numbers (as given by Definition (2.1)) is denoted by $E^{1}$ and is a convex cone. An alternative definition for parameter from of a fuzzy number is given by Kaleva [14].

Definition 2.2. A fuzzy number $\breve{u}$ in parametric form is a pair $(\underline{u}, \bar{u})$ of function $\underline{u}(\alpha), \bar{u}(\alpha), 0 \leq \alpha \leq 1$, which satisfies the following requirement:

i) $\underline{u}(\alpha)$ is a bounded left continuous non- decreasing function over $[0,1]$

ii) $\bar{u}(\alpha)$ is a bounded left continuous non- increasing function over $[0,1]$ 
iii) $\underline{u}(\alpha) \leq \bar{u}(\alpha), 0 \leq \alpha \leq 1$

Definition 2.3. For arbitrary fuzzy $u=(\underline{u}(\alpha), \bar{u}(\alpha)), v=$ $(\underline{v}(\alpha), \bar{v}(\alpha)), 0 \leq \alpha \leq 1$ and scalar $k$, we define addition, subtraction, scalar product by $k$ and multiplication are respectively as following:

1- addition : $(u+v)(\alpha)=(\underline{u}(\alpha)+\underline{v}(\alpha)),(\overline{u+v)}(\alpha)=$ $(\bar{u}(\alpha)+\bar{v}(\alpha))$,

2- subtraction $:(u-v)(\alpha)=(\underline{u}(\alpha)-\underline{v}(\alpha)),(\overline{u-v)}(\alpha)=$ $(\bar{u}(\alpha)-\bar{v}(\alpha))$,

3- scalar product :

$$
k \check{u}=\left\{\begin{array}{l}
(k \underline{u}(\alpha), k \bar{u}(\alpha)), k \geq 0 \\
(k \underline{u}(\alpha), k \bar{u}(\alpha)), k<0
\end{array}\right.
$$

4- multiplication:

$$
\begin{gathered}
\tilde{u} \cdot \tilde{v}= \\
\{\underline{u v}(\alpha)=\max \{\underline{u}(\alpha) \underline{v}(\alpha), \underline{u}(\alpha) \bar{v}(\alpha), \bar{u}(\alpha) \underline{v}(\alpha), \bar{u}(\alpha) \bar{v}(\alpha)\} \\
\overline{\bar{u} v}(\alpha)=\min \{\underline{u}(\alpha) \underline{v}(\alpha), \underline{u}(\alpha) \bar{v}(\alpha), \bar{u}(\alpha) \underline{v}(\alpha), \bar{u}(\alpha) \bar{v}(\alpha)\}
\end{gathered}
$$

Defined 2.4. For arbitrary Fuzzy numbers $\tilde{u}, \tilde{v} \in E^{1}$

$D(\tilde{u}, \tilde{v})=\max \left\{\sup _{\alpha \in[0,1]}|\underline{u}(\alpha)-\underline{v(\alpha)}|, \sup _{\alpha \in[0,1]}|\bar{u}(\alpha)-\bar{v}(\alpha)|\right\}$,

In the distance between The $\widetilde{u}$ and $\tilde{v}$, it is prove $\left(E^{1}, D\right)$ is a complete metric space.

Definition 2.5. The integral of a fuzzy function was define in [14] by using the Riemann integral concept. Let : $[a, b] \rightarrow$ $E^{1}$. For Fuzzy function, for each partition $p=\left\{\mathrm{t}_{0}, \ldots, \mathrm{t}_{\mathrm{n}}\right\}$ of $[a, b]$ and for arbitrary $\xi_{i} \in\left[t_{i-1}, t_{i}\right], 1 \leq i \leq n$, suppose

$$
\begin{gathered}
R_{P}=\sum_{i=1}^{n} f\left(\xi_{i}\right)\left(t_{i}-t_{i-1}\right) \\
\Delta:=\max \left\{\left|t_{i}-t_{i-1}\right|, 1 \leq i \leq n\right\} .
\end{gathered}
$$

The define integral of $f(t)$ over $[a, b]$ is

$$
\begin{aligned}
\underline{u}(x, \alpha) & \left.=\underline{f}(x, \alpha)+\lambda \underline{\int_{a}^{x} k\left(( x , t , F _ { 1 \alpha } ( x , t , u ( t , \alpha ) ) ) \cdot G \left(t, \int_{a}^{t} F_{2 \alpha}(t, s, u(s, \alpha))\right.\right.} d s\right) d t \\
\bar{u}(x, \alpha) & \left.=\bar{f}(x, \alpha)+\lambda \overline{\int_{a}^{x} k\left(( x , t , F _ { 1 \alpha } ( x , t , u ( t , \alpha ) ) ) \cdot G \left(t, \int_{a}^{t} F_{2 \alpha}(t, s, u(s, \alpha))\right.\right.} d s\right) d t
\end{aligned}
$$

let $x, t, s \in[a, b]$

$$
\begin{aligned}
& =\left\{\begin{array}{c}
k\left(\left(x, t, F_{1 \alpha}(t, u(t, \alpha))\right) \cdot G\left(t, \int_{a}^{t} F_{2 \alpha}(t, s, u(s, \alpha)) d s\right)\right. \\
k\left(x, t, \underline{F}_{1 \alpha}(t, \underline{u}(t, \alpha))\right) \cdot G \overline{\left(t, \int_{a}^{t} F_{2 \alpha}(t, s, \underline{u}(s, \alpha)) d s\right), k\left(x, t, F_{1 \alpha}(t, u(t, \alpha))\right) \cdot G\left(t, \int_{a}^{t} F_{2 \alpha}(t, s, u(s, \alpha)) d s\right)} \geq 0 \\
k\left(\left(x, t, \bar{F}_{1 \alpha}(t, \bar{u}(t, \alpha)) \cdot G\left(t, \int_{a}^{t} \bar{F}_{2 \alpha}(t, s, \bar{u}(s, \alpha)) d s\right), \underline{k\left(x, t, F_{1 \alpha}(t, u(t, \alpha))\right) \cdot G\left(t, \int_{a}^{t} F_{2 \alpha}(t, s, u(s, \alpha)) d s\right)}<0\right.\right.
\end{array}\right. \\
& =\left\{\begin{array}{c}
k\left(\left(x, t, F_{1 \alpha}(t, u(t, \alpha))\right) \cdot G\left(t, \int_{a}^{t} F_{2 \alpha}(t, s, u(s, \alpha)) d s\right)\right. \\
k\left(x, t, \underline{F}_{1 \alpha}(t, \underline{u}(t, \alpha))\right) \cdot G \overline{\left(t, \int_{a}^{t} F_{2 \alpha}(t, s, \underline{u}(s, \alpha)) d s\right), k\left(x, t, F_{1 \alpha}(t, u(t, \alpha))\right) \cdot G\left(t, \int_{a}^{t} F_{2 \alpha}(t, s, u(s, \alpha)) d s\right)} \geq 0 \\
k\left(\left(x, t, \bar{F}_{1 \alpha}(t, \bar{u}(t, \alpha)) \cdot G\left(t, \int_{a}^{t} \bar{F}_{2 \alpha}(t, s, \bar{u}(s, \alpha)) d s\right), \underline{k\left(x, t, F_{1 \alpha}(t, u(t, \alpha))\right) \cdot G\left(t, \int_{a}^{t} F_{2 \alpha}(t, s, u(s, \alpha)) d s\right)}<0\right.\right.
\end{array}\right. \\
& \begin{array}{c}
\overline{k\left(x, t, F_{1 \alpha}(x, t, u(t, \alpha))\right) \cdot G\left(t, \int_{a}^{t} F_{2 \alpha}(t, s, u(s, \alpha)) d s\right)}= \\
\left\{\begin{array}{l}
k\left(x, t, \bar{F}_{1 \alpha}(t, \bar{u}(t, \alpha))\right) \cdot G\left(t, \int_{a}^{t} \bar{F}_{2 \alpha}(t, s, \bar{u}(s, \alpha)) d s\right), k \overline{\left(x, t, F_{1 \alpha}(x, t, u(t, \alpha))\right) \cdot G\left(t, \int_{a}^{t} F_{2 \alpha}(t, s, u(s, \alpha)) d s\right)} \geq 0 \\
k\left(x, t, \underline{F}_{1 \alpha}(t, \underline{u}(t, \alpha))\right) \cdot G\left(t, \int_{a}^{t} \underline{F}_{2 \alpha}(t, s, \underline{u}(s, \alpha)) d s\right), \overline{k\left(x, t, F_{1 \alpha}(x, t, u(t, \alpha))\right) \cdot G\left(t, \int_{a}^{t} F_{2 \alpha}(t, s, u(s, \alpha)) d s\right)}<0
\end{array}\right.
\end{array} \\
& \begin{array}{c}
\overline{k\left(x, t, F_{1 \alpha}(x, t, u(t, \alpha))\right) \cdot G\left(t, \int_{a}^{t} F_{2 \alpha}(t, s, u(s, \alpha)) d s\right)}= \\
\left\{\begin{array}{l}
k\left(x, t, \bar{F}_{1 \alpha}(t, \bar{u}(t, \alpha))\right) \cdot G\left(t, \int_{a}^{t} \bar{F}_{2 \alpha}(t, s, \bar{u}(s, \alpha)) d s\right), \overline{k\left(x, t, F_{1 \alpha}(x, t, u(t, \alpha))\right) \cdot G\left(t, \int_{a}^{t} F_{2 \alpha}(t, s, u(s, \alpha)) d s\right)} \geq 0 \\
k\left(x, t, \underline{F}_{1 \alpha}(t, \underline{u}(t, \alpha))\right) \cdot G\left(t, \int_{a}^{t} \underline{F}_{2 \alpha}(t, s, \underline{u}(s, \alpha)) d s\right), \overline{k\left(x, t, F_{1 \alpha}(x, t, u(t, \alpha))\right) \cdot G\left(t, \int_{a}^{t} F_{2 \alpha}(t, s, u(s, \alpha)) d s\right)}<0
\end{array}\right.
\end{array}
\end{aligned}
$$

If the fuzzy function $f(t)$ is continuous in metric $D$, its

It should be noted that the fuzzy integral can be also ed using the Lebesgue - type approach. However, if $f(t)$ is continuous, both approaches yield the same

\section{Proposal Formula for Fuzzy Nonlinear Volterra Integral Equation}

The fuzzy nonlinear integral equation with integral kernel is discussed in this work is the fuzzy nonlinear Volterra integral equation of the second kind (FNVIE-2) as follows:

where $\lambda \geq 0, \tilde{f}(x)$ is a fuzzy function of $\mathrm{x}: \mathrm{a} \leq \mathrm{x} \leq \mathrm{b}$, 列 on $[a, b] \times[a, b] \times[a, b] \times E^{n} \times E^{n}$ and $\tilde{F}_{1}(x, t, \tilde{u}(t))$ $\tilde{F}_{2}(t, s, \tilde{u}(s))$ are nonlinear function on [a, b]. For solving in parametric form of Eq. (7), consider $(\underline{f}(x, \alpha), \bar{f}(x, \alpha))$ and $(\underline{u}(x, \alpha), \bar{u}(x, \alpha)), 0 \leq \alpha \leq 1$ and $\mathrm{t}, \mathrm{s} \in[\mathrm{a}, \mathrm{b}]$ are parametric form of $\tilde{f}(x)$ and $\tilde{u}(x)$, respectively. then, parametric form of Eq. (7) is as follows 
For each $0 \leq \alpha \leq 1$ and $\mathrm{a} \leq \mathrm{x} \leq \mathrm{b}$. We can see that Eq. (7) convert to a system of nonlinear Volterra integral equations in crisp case for each $0 \leq \alpha \leq 1$ and $\mathrm{a} \leq \mathrm{t} \leq \mathrm{b}$. Now, we explain homotopy analysis methods as approximating solution of this system of nonlinear integral equations in crisp case. then, we find approximate solutions for $\tilde{u}(\mathrm{x}), \mathrm{a} \leq \mathrm{x} \leq \mathrm{b}$

\subsection{Generalize Homotopy Analytic Method "HAM"}

Now we apply homotopy analytic method for solve the system (8) and obtain a recursion scheme for it. Prior to apply HAM for the system (8). We suppose that the kernel have four cases for kernal's.

Where

$$
\begin{gathered}
N[\tilde{u}(x, \alpha)]=0 \\
\tilde{u}(x)=\tilde{f}(x)+\lambda \int_{a}^{x} k\left(\left(x, t, \tilde{F}_{1}(x, t, \tilde{u}(t))\right) \cdot G\left(t, \int_{a}^{t} \tilde{F}_{2}(t, s, \tilde{u}(s)) d s\right) d t\right.
\end{gathered}
$$

$$
\tilde{u}(x, \alpha)=(\underline{u}(x, \alpha), \bar{u}(x, \alpha)) \text { and } \tilde{f}(x, \alpha)=(\underline{f}(x, \alpha), \bar{f}(x, \alpha))
$$

We see that eq(7) is convert to system of nonlinear crisp fuzzy volterra integral equations

$$
\begin{aligned}
& \underline{u}(x, \alpha) \underline{f}(x, \alpha)+\lambda{\underline{\int_{a}}}_{k} k\left(\left(x, t, F_{1 \alpha}(x, t, u(t, \alpha))\right) \cdot G\left(t, \int_{a}^{t} F_{2 \alpha}(t, s, u(s, \alpha)) d s\right) d t\right. \\
& \left.\bar{u}(x, \alpha)=\bar{f}(x, \alpha)+\lambda \overline{\int_{a}^{x} k\left(( x , t , F _ { 1 \alpha } ( x , t , u ( t , \alpha ) ) ) \cdot G \left(t, \int_{a}^{t} F_{2 \alpha}(t, s, u(s, \alpha))\right.\right.} d s\right) d t
\end{aligned}
$$

For solving system (9) by HAM, we construct the zeroth-order deformation equations:

$$
\begin{gathered}
(1-p) L\left[\underline{\emptyset}(x, p ; \alpha)-\underline{w}_{0}(x ; \alpha)\right]=p h H[\underline{\emptyset}(x, p ; \alpha)-\underline{f}(x, \alpha) \\
-\lambda \int_{a}^{x} k\left(\left(x, t, \underline{F}_{1 \alpha}(x, t, \underline{\emptyset}(t, p ; \alpha))\right) \cdot G\left(t, \int_{a}^{t} \underline{F}_{2 \alpha}(t, s, \underline{\emptyset}(s, p, \alpha)) d s\right) d t\right. \\
(1-p) L\left[\bar{\varnothing}(x, p ; \alpha)-\bar{w}_{0}(x ; \alpha)\right]=p h H[\bar{\varnothing}(x, p ; \alpha)-\bar{f}(x, \alpha) \\
-\lambda \int_{a}^{x} k\left(\left(x, t, \bar{F}_{1 \alpha}(x, t, \bar{\emptyset}(t, p ; \alpha))\right) \cdot G\left(t, \int_{a}^{t} \bar{F}_{2 \alpha}(t, s, \bar{\emptyset}(s, p, \alpha)) d s\right) d t\right.
\end{gathered}
$$

Where $p \in[a, b]$ is the embedding parameter, his nonzero auxiliary parameter, $L$ is an auxiliary linear operator $H(x)$ is an auxiliary function, $\quad\left(\underline{w}_{0}(x ; \alpha), \bar{w}_{0}(x ; \alpha)\right)$ are initial guesses $\underline{F}_{1 \alpha}(x, t, \underline{\emptyset}(t, p ; \alpha)), \bar{F}_{1 \alpha}(t, s, \bar{\emptyset}(t, p ; \alpha)), \bar{F}_{2 \alpha}(x, t, \bar{\emptyset}(t, p ; \alpha))$ and $\underline{F}_{2 \alpha}(t, s, \underline{\emptyset}(t, p ; \alpha)) \quad$ respectively $\quad$ and $\underline{\emptyset}(t, p ; \alpha)$ and $\bar{\emptyset}(t, p ; \alpha)$ are unknown functions. Using the above zeroth - order deformation equations, with assumption $L(u)=u$ and $H(x)=1$, we have

$$
\begin{gathered}
(1-p)\left[\underline{\emptyset}(x, p ; \alpha)-\underline{w}_{0}(x ; \alpha)\right]=p h[\underline{\emptyset}(x, p ; \alpha)-\underline{f}(x, \alpha) \\
-\lambda \int_{a}^{x} k\left(\left(x, t, \underline{F}_{1 \alpha}(x, t, \underline{\emptyset}(t, p ; \alpha))\right) \cdot G\left(t, \int_{a}^{t} \underline{F}_{2 \alpha}(t, s, \underline{\emptyset}(s, p, \alpha)) d s\right) d t\right. \\
(1-p)\left[\bar{\varnothing}(x, p ; \alpha)-\bar{w}_{0}(x ; \alpha)\right]=p h[\bar{\varnothing}(x, p ; \alpha)-\bar{f}(x, \alpha) \\
-\lambda \int_{a}^{x} k\left(\left(x, t, \bar{F}_{1 \alpha}(x, t, \bar{\emptyset}(t, p ; \alpha))\right) \cdot G\left(t, \int_{a}^{t} \bar{F}_{2 \alpha}(t, s, \bar{\emptyset}(s, p, \alpha)) d s\right) d t\right.
\end{gathered}
$$

Obviously, when $p=0$ and $p=1$ and since $h \neq 0$, we obtain

$$
\begin{gathered}
\underline{\emptyset}(x, 0 ; \alpha)=\underline{w}_{0}(x ; \alpha) \\
\bar{\emptyset}(x, 0 ; \alpha)=\bar{w}_{0}(x ; \alpha)
\end{gathered}
$$

And

$$
\underline{\emptyset}(x, 1 ; \alpha)=\underline{f}(x, \alpha)+\lambda \int_{a}^{x} k\left(\left(x, t, \underline{F_{1 \alpha}}(x, t, \underline{\emptyset}(t, 1 ; \alpha))\right) \cdot G\left(t, \int_{a}^{t} \underline{F}_{2 \alpha}(t, s, \underline{\emptyset}(s, 1, \alpha)) d s\right) d t\right.
$$




$$
\bar{\emptyset}(x, 1 ; \alpha)=\bar{f}(x, \alpha)+\lambda \int_{a}^{x} k\left(\left(x, t, \bar{F}_{1 \alpha}(x, t, \bar{\emptyset}(t, 1 ; \alpha))\right) \cdot G\left(t, \int_{a}^{t} \bar{F}_{2 \alpha}(t, s, \bar{\emptyset}(s, 1, \alpha)) d s\right) d t\right.
$$

Respectively. Thus as $p$ increases from 0 to 1 , the function $\underline{\emptyset}(x, p ; \alpha)$

$\bar{\emptyset}(x, p ; \alpha)$ deforms from the initial guesses $\underline{w}_{0}(x ; \alpha), \bar{w}_{0}(x ; \alpha)$ to the solution of $\underline{F}_{1 \alpha}(x, t, \underline{\emptyset}(t, p ; \alpha)), \bar{F}_{1 \alpha}(t, s, \bar{\varnothing}(t, p ; \alpha)), \bar{F}_{2 \alpha}(x, t, \bar{\varnothing}(t, p ; \alpha))$ and $\underline{F}_{2 \alpha}(t, s, \underline{\emptyset}(t, p ; \alpha))$.

Expanding $\underline{\emptyset}(x, p ; \alpha)$ in Taylors series with respect $p$ gives

$$
\begin{aligned}
& \qquad \underline{\emptyset}(x, p ; \alpha)=\underline{w}_{0}(x ; \alpha)+\sum_{m=1}^{\infty} \underline{u}_{m}(x, \alpha) p^{m} \\
& \bar{\emptyset}(x, p ; \alpha)=\bar{w}_{0}(x ; \alpha)+\sum_{m=1}^{\infty} \bar{u}_{m}(x, \alpha) p^{m}
\end{aligned}
$$

Where

$$
\begin{gathered}
\underline{u}_{m}(x, r)=\left.\frac{1}{m !} \frac{\partial^{m} \underline{\emptyset}(t, p ; r)}{\partial p^{m}}\right|_{p=0} \\
\bar{u}_{m}(x, r)=\left.\frac{1}{m !} \frac{\partial^{m} \bar{\emptyset}(t, p ; r)}{\partial p^{m}}\right|_{p=0}, m \geq 1
\end{gathered}
$$

It should be noted that $\underline{\emptyset}(x, p ; \alpha)=\underline{w}_{0}(x ; \alpha)$ and $\bar{\emptyset}(x, p ; \alpha)=\bar{w}_{0}(x ; \alpha)$ differential the zeroth - order deformation equation (11) $m$ times with respect to the embedding parameter $p$ and dividing them by $m$ ! and $n$ ! finally setting $p=0$,we obtain the so called $m-t h$ order deformation equations

$$
\begin{array}{r}
\underline{u}_{m}(x ; r)=h\left[\underline{u}_{m-1}(x ; r)-\left(1-x_{m}\right) \underline{f}(x ; r)-\lambda \int_{a}^{x} k\left(\left(x, t, \underline{F}_{1 \alpha}\left(x, t, \underline{\emptyset_{m-1}}(t, 1 ; \alpha)\right)\right) \cdot G\left(t, \int_{a}^{t} \underline{F}_{2 \alpha}\left(t, s, \underline{\emptyset_{m-1}}(s, 1, \alpha)\right) d s\right) d t\right.\right. \\
\bar{u}_{m}(x ; r)=h\left[\bar{u}_{m-1}(x ; r)-\left(1-x_{m}\right) \bar{f}(x ; r)--\lambda \int_{a}^{x} k\left(\left(x, t, \bar{F}_{1 \alpha}\left(x, t, \bar{\emptyset}_{m-1}(t, p ; \alpha)\right)\right) G\left(t, \int_{a}^{t} \bar{F}_{2 \alpha}\left(t, s, \overline{\emptyset_{m-1}}(s, p, \alpha)\right) d s\right) d t(14)\right.\right.
\end{array}
$$

Where

$$
\begin{aligned}
& x_{m}=\left\{\begin{array}{l}
0, m=1 \\
1, m \neq 1
\end{array} m \geq 1\right. \\
& \underline{\emptyset}(x, \alpha)=\underline{w}_{0}(x ; \alpha) \text {, and } \\
& \underline{R}_{m-1}(x, t ; \alpha)=\left.\frac{1}{(m-1) !} \frac{\partial^{m-1} k\left(x, t, \underline{F}_{1 \alpha}(x, \underline{\emptyset}(x, p ; \alpha)) \cdot G\left(t, \int_{a}^{t} \underline{F}_{2 \alpha}(t, s, \underline{\emptyset}(s, p, \alpha) d s)\right.\right.}{\partial p^{m-1}}\right|_{p=0}
\end{aligned}
$$

and

$$
\begin{gathered}
\bar{\emptyset}(x, \alpha)=\bar{w}_{0}(x ; \alpha) \\
\bar{R}_{m-1}(x, t ; \alpha)=\left.\frac{1}{(m-1) !} \frac{\partial^{m-1} k\left(x, t, \bar{F}_{1 \alpha}(x, \bar{\Phi}(x, p ; \alpha))\right) \cdot G\left(t, \int_{a}^{t} \bar{F}_{2 \alpha}(t, s, \bar{\emptyset}(s, p, \alpha) d s)\right.}{\partial p^{m-1}}\right|_{p=0}
\end{gathered}
$$

From Eqs (14) and (15), we have that

$$
\begin{gathered}
\underline{u}_{0}(x, \alpha)=0 \\
\underline{u}_{1}(x ; \alpha)=h \underline{u}_{0}(x ; \alpha)-h \underline{f}(x ; \alpha)-\lambda h\left[\int_{0}^{x} \underline{R}_{0}(x, t ; \alpha) d t\right] \\
\underline{u}_{m}(x ; \alpha)=(1+h) \underline{u}_{m-1}(x ; \alpha)-\lambda h\left[\int_{0}^{x} \underline{R}_{m-1}(x, t ; \alpha) d t\right]
\end{gathered}
$$

Similary

$$
\bar{u}_{0}(x, \alpha)=0
$$




$$
\begin{gathered}
\bar{u}_{1}(x ; \alpha)=h \underline{u}_{0}(x ; \alpha)-h \underline{f}(x ; \alpha)-\lambda h\left[\int_{0}^{x} \underline{R}_{0}(x, t ; \alpha) d t\right] \\
\bar{u}_{m}(x ; \alpha)=(1+h) \underline{u}_{m-1}(x ; \alpha)-\lambda h\left[\int_{0}^{x} \underline{R}_{m-1}(x, t ; \alpha) d t\right]
\end{gathered}
$$

where,

$$
\begin{aligned}
& \underline{R}_{0}(x, t ; \alpha)=k\left(x, t, \underline{F}_{1 \alpha}\left(t, \underline{u_{0}}(t, \alpha)\right)\right) \cdot G\left(t, \int_{a}^{t} \underline{F}_{2 \alpha}\left(t, s, \underline{u}_{0}(s, \alpha)\right) d s\right) \\
& \bar{R}_{0}(x, t ; \alpha)=k\left(x, t, \bar{F}_{1 \alpha}\left(t, \overline{u_{0}}(t, \alpha)\right)\right) \cdot G\left(t, \int_{a}^{t} \bar{F}_{2 \alpha}\left(t, s, \bar{u}_{0}(s, \alpha)\right) d s\right)
\end{aligned}
$$

Thus, if we choose a proper values of $h$, the series (9) is convergent at $p=1$. Hence the solution of system (10) in series from (homotopy solution series) is obtain as

$$
\begin{aligned}
& \underline{U}(x, \alpha)=\underline{u}_{0}(x ; \alpha)+\sum_{m=1}^{\infty} \underline{u}_{m}(x ; \alpha) \\
& \bar{U}(x, \alpha)=\bar{u}_{0}(x ; \alpha)+\sum_{m=1}^{\infty} \bar{u}_{m}(x ; \alpha)
\end{aligned}
$$

We denoted the $n-t h$ order approximate solution with

$$
\begin{gathered}
\underline{U_{j}}(x, r)=\underline{u}_{0}(x ; r)+\sum_{m=1}^{\infty} \underline{u}_{m}(x ; r) \\
\overline{U_{j}}(x, r)=\bar{u}_{0}(x ; r)+\sum_{m=1}^{\infty} \bar{u}_{m}(x ; r) \\
j \geq 1
\end{gathered}
$$

\subsection{Existence and Convergence Analysis}

Consider $\tilde{f}(x)$ is a bounded $\forall x \in[a, b]$ and

$$
|\mathrm{k}(\mathrm{x}, \mathrm{t})| \leq \Re, \forall a \leq x, t, \leq b
$$

We also suppose the nonlinear operators $\tilde{F}_{1}(x, t, \tilde{u}(t)), \tilde{F}_{2}(t, s, \tilde{u}(s))$ are satisfied in Lipschitz condition

$$
\begin{aligned}
& D\left(\tilde{F}_{1}(x, t, \tilde{u}(t))-\tilde{F}_{1}\left(x, t, \tilde{u}^{*}(t)\right)\right) \leq L L_{1} D\left(\tilde{u}, \tilde{u}^{*}\right) \\
& D\left(\tilde{F}_{2}(t, s, \tilde{u}(s))-\tilde{F}_{2}\left(t, s, \tilde{u}^{*}(s)\right)\right) \leq L^{*} L_{1}^{*} D\left(\tilde{u}, \tilde{u}^{*}\right)
\end{aligned}
$$

Let

$$
\alpha=\lambda\left(L L_{1} \Re+L^{*} L_{1}^{*} \Re^{*}\right)
$$

Now we will prove the existence and uniqueness and convergence of the solution, of the method by using Homotopy analysis method.

Theorem 3.2.1:

Let $0<\alpha<1$, then equation (7) has a unique solution.

Proof: Let $u$ and $u^{*}$ be two different solutions of equation (7) then

$$
\begin{gathered}
D\left(\tilde{u}, \tilde{u}^{*}\right)=D\left(\tilde{f}(x)+\lambda \int_{a}^{x} k\left(\left(x, t, \tilde{F}_{1}(x, t, \tilde{u}(t))\right) \cdot G\left(t, \int_{a}^{t} \tilde{F}_{2}(t, s, \tilde{u}(s)) d s\right) d t\right.\right. \\
\tilde{f}(x)+\lambda \int_{a}^{x} k\left(\left(x, t, \tilde{F}_{1}\left(x, t, \tilde{u}^{*}(t)\right)\right) \cdot G\left(t, \int_{a}^{t} \tilde{F}_{2}\left(t, s, \tilde{u}^{*}(s)\right) d s\right) d t\right) \\
D\left(\tilde{u}, \tilde{u}^{*}\right)=\max \left\{\sup \mid \tilde{f}(x)+\lambda \int_{a}^{x} k\left(\left(x, t, \tilde{F}_{1}(x, t, \tilde{u}(t))\right) \cdot G\left(t, \int_{a}^{t} \tilde{F}_{2}(t, s, \tilde{u}(s)) d s\right) d t\right.\right.
\end{gathered}
$$




$$
\begin{aligned}
& -\tilde{f}(x)+\lambda \int_{a}^{x} k\left(\left(x, t, \tilde{F}_{1}\left(x, t, \tilde{u}^{*}(t)\right)\right) \cdot G\left(t, \int_{a}^{t} \tilde{F}_{2}\left(t, s, \tilde{u}^{*}(s)\right) d s\right) d t \mid,\right. \\
& \sup \mid \tilde{f}(x)+\lambda \int_{a}^{x} k\left(\left(x, t, \tilde{F}_{1}\left(x, t, \tilde{u}^{*}(t)\right)\right) \cdot G\left(t, \int_{a}^{t} \tilde{F}_{2}\left(t, s, \tilde{u}^{*}(s)\right) d s\right) d t\right. \\
& -\tilde{f}(x)+\lambda \int_{a}^{x} k\left(\left(x, t, \tilde{F}_{1}(x, t, \tilde{u}(t))\right) \cdot G\left(t, \int_{a}^{t} \tilde{F}_{2}(t, s, \tilde{u}(s)) d s\right) d t\right. \\
& \leq \max \left\{\operatorname { s u p } \lambda \left(\int_{a}^{x} \mid k\left(\left(x, t, \tilde{F}_{1}(x, t, \tilde{u}(t))\right) \cdot G\left(t, \int_{a}^{t} \tilde{F}_{2}(t, s, \tilde{u}(s)) d s\right) d t\right.\right.\right. \\
& -\int_{a}^{x} k\left(\left(x, t, \tilde{F}_{1}\left(x, t, \tilde{u}^{*}(t)\right)\right) \cdot G\left(t, \int_{a}^{t} \tilde{F}_{2}\left(t, s, \tilde{u}^{*}(s)\right) d s\right) d t \mid\right), \\
& \sup \lambda\left(\int_{a}^{x} \mid k\left(\left(x, t, \tilde{F}_{1}\left(x, t, \tilde{u}^{*}(t)\right)\right) \cdot G\left(t, \int_{a}^{t} \tilde{F}_{2}\left(t, s, \tilde{u}^{*}(s)\right) d s\right) d t \mid\right.\right. \\
& -\int_{a}^{x} k\left(\left(x, t, \tilde{F}_{1}(x, t, \tilde{u}(t))\right) \cdot G\left(t, \int_{a}^{t} \tilde{F}_{2}(t, s, \tilde{u}(s)) d s\right) d t \mid\right) \\
& \leq \max \left\{\operatorname { s u p } \lambda \left(\int_{a}^{x} \mid k\left(\left(x, t, \tilde{F}_{1}(x, t, \tilde{u}(t))\right) \cdot G\left(t, \int_{a}^{t} \tilde{F}_{2}(t, s, \tilde{u}(s)) d s\right) d t\right.\right.\right. \\
& -k\left(\left(x, t, \tilde{F}_{1}(x, t, \tilde{u}(t))\right) \cdot G\left(t, \int_{a}^{t} \tilde{F}_{2}\left(t, s, \tilde{u}^{*}(s)\right) d s\right)+k\left(\left(x, t, \tilde{F}_{1}(x, t, \tilde{u}(t))\right) \cdot G\left(t, \int_{a}^{t} \tilde{F}_{2}\left(t, s, \tilde{u}^{*}(s)\right) d s\right)\right.\right. \\
& -k\left(\left(x, t, \tilde{F}_{1}\left(x, t, \tilde{u}^{*}(t)\right)\right) \cdot G\left(t, \int_{a}^{t} \tilde{F}_{2}\left(t, s, \tilde{u}^{*}(s)\right) d s\right) d t \mid\right\}, \\
& \sup \lambda\left(\int_{a}^{x} \mid k\left(\left(x, t, \tilde{F}_{1}\left(x, t, \tilde{u}^{*}(t)\right)\right) \cdot G\left(t, \int_{a}^{t} \tilde{F}_{2}\left(t, s, \tilde{u}^{*}(s)\right) d s\right) d t\right.\right. \\
& -k\left(\left(x, t, \tilde{F}_{1}\left(x, t, \tilde{u}^{*}(t)\right)\right) \cdot G\left(t, \int_{a}^{t} \tilde{F}_{2}(t, s, \tilde{u}(s)) d s\right) d t\right. \\
& +k\left(\left(x, t, \tilde{F}_{1}\left(x, t, \tilde{u}^{*}(t)\right)\right) \cdot G\left(t, \int_{a}^{t} \tilde{F}_{2}(t, s, \tilde{u}(s)) d s\right) d t\right. \\
& -k\left(\left(x, t, \tilde{F}_{1}(x, t, \tilde{u}(t))\right) \cdot G\left(t, \int_{a}^{t} \tilde{F}_{2}(t, s, \tilde{u}(s)) d s\right) d t \mid\right\} \\
& \leq \max \left\{\sup \mid \lambda \int_{a}^{x} k\left(\left(x, t, \tilde{F}_{1}(x, t, \tilde{u}(t))\right) d t|.| G\left(t, \int_{a}^{t} \tilde{F}_{2}(t, s, \tilde{u}(s)) d s\right)-\right.\right. \\
& \left|G\left(t, \int_{a}^{t} \tilde{F}_{2}\left(t, s, \tilde{u}^{*}(s)\right) d s\right)\right|+\left|G\left(t, \int_{a}^{t} \tilde{F}_{2}\left(t, s, \tilde{u}^{*}(s)\right) d s\right)\right| \cdot \mid k\left(\left(x, t, \tilde{F}_{1}(x, t, \tilde{u}(t))\right)-k\left(\left(x, t, \tilde{F}_{1}\left(x, t, \tilde{u}^{*}(t)\right)\right) \mid,\right.\right. \\
& \sup \mid \lambda \int_{a}^{x} k\left(\left(x, t, \tilde{F}_{1}\left(x, t, \tilde{u}^{*}(t)\right)\right)|\cdot| G\left(t, \int_{a}^{t} \tilde{F}_{2}\left(t, s, \tilde{u}^{*}(s)\right) d s\right)-G\left(t, \int_{a}^{t} \tilde{F}_{2}(t, s, \tilde{u}(s)) d s\right) \mid+\right. \\
& \left|G\left(t, \int_{a}^{t} \tilde{F}_{2}(t, s, \tilde{u}(s)) d s\right)\right| \cdot \mid k\left(\left(x, t, \tilde{F}_{1}\left(x, t, \tilde{u}^{*}(t)\right)-k\left(\left(x, t, \tilde{F}_{1}(x, t, \tilde{u}(t))\right) \mid\right\}\right.\right. \\
& \leq \lambda \sup _{a<x<b, 0<t<1} \mid D\left(k\left(\left(x, t, \tilde{F}_{1}(x, t, \tilde{u}(t))\right), \tilde{0}\right)|\cdot| \int_{a}^{x} G\left(t, \int_{a}^{t} \widetilde{F}_{2}(t, s, \widetilde{u}(s)) d s\right) d t-\int_{a}^{x} G\left(t, \int_{a}^{t} \widetilde{F}_{2}\left(t, s, \tilde{u}^{*}(s)\right) d s\right) d t \mid\right. \\
& +\left|\int_{a}^{x} G\left(t, \int_{a}^{t} \tilde{F}_{2}\left(t, s, \tilde{u}^{*}(s)\right) d s\right) d t\right| . D \mid\left(\left(x, t, \tilde{F}_{1}(x, t, \tilde{u}(t))\right)-k\left(\left(x, t, \tilde{F}_{1}\left(x, t, \tilde{u}^{*}(t)\right)\right) \mid,\right.\right.
\end{aligned}
$$




$$
\begin{aligned}
& \lambda \sup _{a<x<b, 0<t<1} D \mid\left(k \left(\left(x, t, \tilde{F}_{1}\left(x, t, \tilde{u}^{*}(t)\right), \tilde{0}\right)|.| \int_{a}^{x} G\left(t, \int_{a}^{t} \widetilde{F}_{2}\left(t, s, \tilde{u}^{*}(s)\right) d s\right) d t\left|-\int_{a}^{x} G\left(t, \int_{a}^{t} \tilde{F}_{2}(t, s, \tilde{u}(s)) d s\right) d t\right|\right.\right. \\
& \quad+\left|\int_{a}^{x} G\left(t, \int_{a}^{t} \widetilde{F}_{2}(t, s, \widetilde{u}(s)) d s\right) d t\right| \cdot D \mid k\left(\left(x, t, \widetilde{F}_{1}\left(x, t, \widetilde{u}^{*}(t)\right)-k\left(\left(x, t, \tilde{F}_{1}(x, t, \tilde{u}(t))\right) \mid\right.\right.\right. \\
& \leq \lambda \sup \left(\int_{a}^{x} D\left(\tilde{F}_{1}(x, t, \tilde{u}(t)), \tilde{0}\right) \cdot L\left(\int_{a}^{x} D\left(\widetilde{F}_{2}(t, s, \widetilde{u}(s))-\tilde{F}_{2}\left(t, s, \tilde{u}^{*}(s)\right)\right) d s\right)+\left(\int_{a}^{x} D\left(\tilde{F}_{2}\left(t, s, \tilde{u}^{*}(s)\right)\right) d s, \tilde{0}\right) .\right. \\
& L_{1}\left(\widetilde{F}_{1}(x, t, \tilde{u}(t))-\widetilde{F}_{1}\left(x, t, \widetilde{u}^{*}(t)\right)\right), \lambda \sup \left(D ( \widetilde { F } _ { 1 } ( x , t , \widetilde { u } ^ { * } ( t ) ) , \widetilde { 0 } ) \cdot L ^ { * } \left(\int_{a}^{x} D\left(\tilde{F}_{2}\left(t, s, \tilde{u}^{*}(s)\right)-\widetilde{F}_{2}(t, s, \widetilde{u}(s)) d s\right)\right.\right. \\
& \quad+\left(\int_{a}^{x} D\left(\widetilde{F}_{2}(t, s, \tilde{u}(s)) d s\right) \cdot L_{1}^{*}\left(\tilde{F}_{1}(x, t, \tilde{u}(t))-\widetilde{F}_{1}\left(x, t, \tilde{u}^{*}(t)\right)\right)\right.
\end{aligned}
$$

$\leq \lambda \sup D\left(\tilde{F}_{1}(x, t, \tilde{u}(t)), \tilde{0}\right) \cdot L L_{1}\left(\tilde{F}_{2}\left(t, s, \tilde{u}^{*}(s)\right)-\widetilde{F}_{2}(t, s, \widetilde{u}(s))\right), \lambda \sup D\left(\tilde{F}_{2}\left(t, s, \tilde{u}^{*}(s)\right), \tilde{0}\right) \cdot L^{*} L_{1}^{*}\left(\tilde{F}_{1}(x, t, \tilde{u}(t))-\widetilde{F}_{1}\left(x, t, \widetilde{u}^{*}(t)\right)\right)$

$$
\begin{gathered}
\leq \lambda \sup L L_{1} D\left(\tilde{F}_{1}(x, t, \tilde{u}(t)), \tilde{0}\right) \cdot D\left(\tilde{u}, \tilde{u}^{*}\right), \lambda \sup L^{*} L_{1}^{*} D\left(\tilde{F}_{2}(x, t, \tilde{u}(t)), \tilde{0}\right) \cdot D\left(\tilde{u}, \tilde{u}^{*}\right) \\
\leq \lambda \sup (b-a) L L_{1} D\left(\tilde{F}_{1}(x, t, \tilde{u}(t)), \tilde{0}\right) \cdot D\left(\tilde{u}, \tilde{u}^{*}\right), \lambda \sup (b-a) L^{*} L_{1}^{*} D\left(\tilde{F}_{2}(x, t, \tilde{u}(t)), \tilde{0}\right) \cdot D\left(\tilde{u}, \tilde{u}^{*}\right) \\
\leq \lambda(b-a) L L_{1} D\left(\tilde{F}_{1}(x, t, \tilde{u}(t)), \tilde{0}\right) \cdot D\left(\tilde{u}, \tilde{u}^{*}\right), \lambda(b-a) L^{*} L_{1}^{*} D\left(\tilde{F}_{2}(x, t, \tilde{u}(t)), \tilde{0}\right) \cdot D\left(\tilde{u}, \tilde{u}^{*}\right)
\end{gathered}
$$

Then we let $D\left(\tilde{F}_{1}(x, t, \tilde{u}(t)), \tilde{0}\right)=\Re$ and $D\left(\tilde{F}_{2}(x, t, \tilde{u}(t)), \tilde{0}\right)=\Re^{*}$

We get

$$
\leq \lambda\left(L L_{1} \Re+L^{*} L_{1}^{*} \Re^{*}\right)(b-a)^{2} D\left(\tilde{u}, \tilde{u}^{*}\right)
$$

let

$$
\alpha=\lambda\left(L L_{1} \Re+L^{*} L_{1}^{*} \Re^{*}\right)=\alpha D\left(\tilde{u}, \tilde{u}^{*}\right)
$$

From which we get $(1-\alpha) D\left(\tilde{u}, \tilde{u}^{*}\right) \leq 0$. Since $0<\alpha<1$, then $D\left(\tilde{u}, \tilde{u}^{*}\right)=0$. Implies $\tilde{u}=\tilde{u}^{*}$

Theorem 3.2.2. The series solution $\bar{u}(x, \alpha)=\sum_{i=0}^{\infty} \bar{u}_{i}(x, \alpha)$ of equation (7) using HAM convergence when $0<\alpha<$ $1,\left|\bar{u}_{i}(x, \alpha)\right|<\infty$

Proof: The series of partition sum $\bar{T}_{n}$, let $\bar{T}_{n}$ and $\bar{T}_{m}$ be arbitrary partial sums with $n \geq m$. We are going to prove that $\bar{T}_{n}$ is a Cauchy sequence in this banach space

$$
\begin{gathered}
|| \bar{T}_{n}-\bar{T}_{m} \|=\max _{\forall x \in[a, b]} \mid \lambda\left[\int _ { a } ^ { x } k \left(\left(x, t, \widetilde{F}_{1}\left(x, t, \widetilde{u}_{n-1}(t)\right)\right) \cdot G\left(t, \int_{a}^{t} \widetilde{F}_{2}\left(t, s, \widetilde{u}_{n-1}(s)\right) d s\right) d t\right.\right. \\
\quad-\int_{a}^{x} k\left(\left(x, t, \widetilde{F}_{1}\left(x, t, \widetilde{u}_{m-1}(t)\right)\right) \cdot G\left(t, \int_{a}^{t} \widetilde{F}_{2}\left(t, s, \widetilde{u}_{m-1}(s)\right) d s\right) d t\right] \mid \\
\leq \int_{a}^{x} \mid k\left(\left(x, t, \tilde{F}_{1}\left(x, t, \tilde{u}_{n-1}(t)\right)\right) \cdot G\left(t, \int_{a}^{t} \tilde{F}_{2}\left(t, s, \tilde{u}_{n-1}(s)\right) d s\right) d t-k\left(\left(x, t, \tilde{F}_{1}\left(x, t, \tilde{u}_{n-1}(t)\right) G\left(t, \int_{a}^{t} \tilde{F}_{2}\left(t, s, \tilde{u}_{m-1}(s)\right) d s\right.\right.\right.\right. \\
+k\left(\left(x, t, \tilde{F}_{1}\left(x, t, \tilde{u}_{n-1}(t)\right) G\left(t, \int_{a}^{t} \tilde{F}_{2}\left(t, s, \tilde{u}_{m-1}(s)\right) d s\right.\right.\right. \\
+\int_{a}^{x} k\left(\left(x, t, \tilde{F}_{1}\left(x, t, \tilde{u}_{m-1}(t)\right)\right) \cdot G\left(t, \int_{a}^{t} \tilde{F}_{2}\left(t, s, \tilde{u}_{m-1}(s)\right) d s\right) d t\right] \mid, \\
\int_{a}^{x} \mid k\left(\left(x, t, \tilde{F}_{1}\left(x, t, \tilde{u}_{m-1}(t)\right)\right) \cdot G\left(t, \int_{a}^{t} \tilde{F}_{2}\left(t, s, \tilde{u}_{m-1}(s)\right) d s\right) d t-k\left(\left(x, t, \tilde{F}_{1}\left(x, t, \tilde{u}_{m-1}(t)\right) G\left(t, \int_{a}^{t} \tilde{F}_{2}\left(t, s, \tilde{u}_{n-1}(s)\right) d s\right.\right.\right.\right. \\
+k\left(\left(x, t, \tilde{F}_{1}\left(x, t, \tilde{u}_{m-1}(t)\right) G\left(t, \int_{a}^{t} \tilde{F}_{2}\left(t, s, \tilde{u}_{n-1}(s)\right) d s+k\left(( x , t , \tilde { F } _ { 1 } ( x , t , \tilde { u } _ { n - 1 } ( t ) ) ) \cdot G \left(t, \int_{a}^{t} \tilde{F}_{2}\left(t, s, \tilde{u}_{n-1}(s)\right) d s \mid\right.\right.\right.\right.\right.
\end{gathered}
$$

by using the theorem 3.2.1 we get

$$
\leq \alpha|| \bar{T}_{n}-\bar{T}_{m}||
$$

Let $\mathrm{n}=\mathrm{m}+1$, then 


$$
|| \bar{T}_{n}-\bar{T}_{m}\left\|\leq \alpha|| \bar{T}_{m}-\bar{T}_{m-1}\right\| \leq \alpha^{2}|| \bar{T}_{m-1}-\bar{T}_{m-2}|| \ldots \ldots \ldots \ldots \ldots \leq \alpha^{m}|| \bar{T}_{1}-\bar{T}_{0} \|
$$

We have,

$$
\begin{gathered}
|| \bar{T}_{n}-\bar{T}_{m}\left\|\leq \leq|| \bar{T}_{m+1}-\bar{T}_{m}\right\|+|| \bar{T}_{m+2}-\bar{T}_{m+1}||+\cdots \ldots \ldots \ldots+|| \bar{T}_{n}-\bar{T}_{n-1} \| \\
\leq\left[\alpha^{m}+\alpha^{m+1}+\cdots \ldots \ldots .+\alpha^{m-1}\right]|| \bar{T}_{1}-\bar{T}_{0} \| \\
\leq \alpha^{m}\left[1+\alpha+\alpha^{2}+\cdots \ldots \ldots .+\alpha^{n-m-1}\right]|| \bar{T}_{1}-\bar{T}_{0} \| \\
\quad \leq \alpha^{m}\left[\frac{1-\alpha^{n-m}}{1-\alpha}\right]\left\|\bar{u}_{1}(x, \alpha)\right\|
\end{gathered}
$$

Since $0<\alpha<1$, we have $\left(1-\alpha^{n-m}\right)<1$, then

$$
|| \bar{T}_{n}-\bar{T}_{m}|| \leq \frac{\alpha^{m}}{1-\alpha} \max _{\forall t}\left|\bar{u}_{1}(x, \alpha)\right|
$$

But $\left|\bar{u}_{1}(x, \alpha)\right|<\infty$, as $m \rightarrow \infty$, then || $\bar{T}_{n}-\bar{T}_{m} \| \rightarrow 0$. We conclude that $\bar{T}_{n}$ is Cauchy sequence

$$
\bar{u}(x, \alpha)=\lim _{n \rightarrow \infty} \bar{u}_{n}(x, \alpha)
$$

Similarly, we have $\underline{T}_{n}$ is a Cauchy sequence, then we have

$$
\underline{u}(x, \alpha)=\lim _{n \rightarrow \infty} \underline{u}_{n}(x, \alpha)
$$

Finally we will have

$$
\tilde{u}(x, \alpha)=\lim _{n \rightarrow \infty} \tilde{u}_{n}(x, \alpha)
$$

Theorem 3.2.3: fuzzy nonlinear Volterra integral equation is convergent to the exact solution when using Homotopy analysis method.

Proof: for $n \geq 0$

$$
\begin{gathered}
\vartheta_{n+1}(x, t)=\tilde{f}(x)+\sum_{i=1}^{n+1}\left[\lambda \int_{a}^{x} k\left(\left(x, t, \tilde{F}_{1}\left(x, t, \tilde{u}_{i}(t)\right)\right) \cdot G\left(t, \int_{a}^{t} \tilde{F}_{2}\left(t, s, \tilde{u}_{\imath}(s)\right) d s\right) d t\right]\right. \\
D\left(\vartheta_{n+1}(x, t), \vartheta_{n}(x, t)\right) \\
=D\left(\vartheta_{n}(x, t)+\lambda \int_{a}^{x} k\left(\left(x, t, \tilde{F}_{1}\left(x, t, \tilde{u}_{n+1}(t)\right)\right) \cdot G\left(t, \int_{a}^{t} \tilde{F}_{2}\left(t, s, \widetilde{u_{n+1}}(s)\right) d s\right) d t, \vartheta_{n}(x, t)\right)\right. \\
=D\left(\lambda \int_{a}^{x} k\left(\left(x, t, \tilde{F}_{1}\left(x, t, \tilde{u}_{n+1}(t)\right)\right) \cdot G\left(t, \int_{a}^{t} \tilde{F}_{2}\left(t, s, \widetilde{u_{n+1}}(s)\right) d s\right) d t, \tilde{0}\right) \leq D\left(\tilde{u}_{n+1}(x), \tilde{0}\right)\right. \\
D\left(\tilde{u}_{n}(x), \tilde{0}\right) \leq \alpha^{n} D(\tilde{f}, \tilde{0})
\end{gathered}
$$

So

$$
D\left(\vartheta_{n+1}, \vartheta_{n}\right) \leq \alpha^{n+1} D(\tilde{f}, \tilde{0})
$$

Then

$$
\sum_{n=0}^{\infty} D\left(\vartheta_{n+1}, \vartheta_{n}\right) \leq \alpha^{n+1} D(\tilde{f}, \tilde{0}) \sum_{n=0}^{\infty} \alpha^{n}, 0<\alpha<1
$$

\subsection{Numerical Example}

In this part we will discuss all of cases for our formula by using homotopy analysis method. And comparing the approximate 
method with he known exact solution and calculate the absolute error between the exact and approximate. Also give some finger for all cases

Example 1:

Now we discuss this example by using HAM

$$
\begin{aligned}
& \underline{u}(x, \alpha)=\underline{f}(x, \alpha)+\lambda \int_{a}^{x} k\left(x, t, \underline{F}_{1 \alpha}(x, t, \underline{u}(t, \alpha))\right) \cdot G\left(t, \int_{a}^{t} \underline{F}_{2 \alpha}(t, s, \underline{u}(s, \alpha)) d s\right) d t \\
& \bar{u}(x, \alpha)=\bar{f}(x, \alpha)+\lambda \int_{a}^{x} k\left(x, t, \bar{F}_{1 \alpha}(x, t, \bar{u}(t, \alpha))\right) \cdot G\left(t, \int_{a}^{t} \bar{F}_{2 \alpha}(t, s, \bar{u}(s, \alpha)) d s\right) d t
\end{aligned}
$$

where

$$
\begin{aligned}
k\left(\left(x, t, \underline{F}_{1 \alpha}(t, \underline{u}(t, \alpha))\right) \cdot G\left(t, \int_{a}^{t} \underline{F}_{2 \alpha}(t, s, \underline{u}(s, \alpha)) d s\right)\right. & =x t(t, \underline{u}(t, \alpha))^{2} \cdot G\left(t, \int_{a}^{t}(t, s, \underline{u}(s, \alpha))^{3} d s\right) \\
k\left(\left(x, t, \bar{F}_{1 \alpha}(t, \bar{u}(t, \alpha))\right) \cdot G\left(t, \int_{a}^{t} \bar{F}_{2 \alpha}(t, s, \bar{u}(s, \alpha)) d s\right)\right. & =x t(t, \bar{u}(t, \alpha))^{3} \cdot G\left(t, \int_{a}^{t}(t, s, \bar{u}(s, \alpha))^{2} d s\right) \\
\underline{F}_{1 \alpha}(x, u(x, \alpha)) & =(\underline{u}(x, \alpha))^{2} \\
\underline{F}_{2 \alpha}(x, u(x, \alpha)) & =(\underline{u}(x, \alpha))^{3} \\
\bar{F}_{1 \alpha}(x, u(x, \alpha)) & =(\bar{u}(x, \alpha))^{2} \\
\bar{F}_{2 \alpha}(x, u(x, \alpha)) & =(\bar{u}(x, \alpha))^{3}
\end{aligned}
$$

the intimal condition is $\underline{u}_{0}(x, \alpha)=\bar{u}_{0}(x, \alpha)=0$, and $0 \leq \alpha \leq 1$

the exact solution $\underline{u}(x, \alpha)=x \alpha$ and $\bar{u}(x, \alpha)=x(2-\alpha)$

$$
\begin{gathered}
\underline{f}(x, \alpha)=x * r-(1 / 32) * x^{\wedge} 9 * r^{\wedge} 5 \\
\bar{f}(x, \alpha)=x *(2-r)-(1 / 32) * x^{\wedge} 9 *(2-r)^{\wedge} 5
\end{gathered}
$$

Now we will use the homotopy analysis method to solve the example 1 we can see the first term of this method

$$
\begin{aligned}
& \underline{u}_{0}(x, \alpha)=0 \\
& \underline{u}_{1}(x, \alpha)=-h *\left(x * r-(1 / 32) * x^{\wedge} 9 * r^{\wedge} 5\right) \\
& \underline{u}_{2}(x, \alpha)=-(1+h) * h *\left(x * r-(1 / 32) * x^{\wedge} 9 * r^{\wedge} 5\right)-h *\left(-h^{\wedge} 5 *\left(-(1 / 45097156608) * r^{\wedge} 25 * x^{\wedge} 49\right.\right. \\
& +(31 / 5872025600) * r^{\wedge} 21 * x^{\wedge} 41-(41 / 73400320) * r^{\wedge} 17 * x^{\wedge} 33+(3 / 81920) * r^{\wedge} 13 * x^{\wedge} 25 \\
& \left.\left.-(3 / 2048) * r^{\wedge} 9 * x^{\wedge} 17+(1 / 32) * x^{\wedge} 9 * r^{\wedge} 5\right)\right)
\end{aligned}
$$

where

$$
\underline{R}_{0}(x, t, \alpha)=x t\left(t, \underline{u_{0}}(t, \alpha)\right)^{2} \cdot G\left(t, \int_{a}^{t}\left(t, s, \underline{u_{0}}(s, \alpha)\right)^{3} d s\right)
$$

$\underline{u}_{3}(x, \alpha)=(1+\mathrm{h}) *\left(-(1+\mathrm{h}) \mathrm{h}\left(\mathrm{x} \mathrm{r}-1 / 32 \mathrm{x}^{\wedge} 9 \mathrm{r}^{\wedge} 5\right)+\mathrm{h}^{\wedge} 6\left(-1 / 45097156608 \mathrm{r}^{\wedge} 25 \mathrm{x}^{\wedge} 49+31 / 5872025600 \mathrm{r}^{\wedge} 21 \mathrm{x}^{\wedge} 41-41 / 73400320 \mathrm{r}^{\wedge} 17\right.\right.$ $\left.\left.\mathrm{x}^{\wedge} 33+3 / 81920 \quad \mathrm{r}^{\wedge} 13 \quad \mathrm{x}^{\wedge} 25-3 / 2048 \quad \mathrm{r}^{\wedge} 9 \quad \mathrm{x}^{\wedge} 17+1 / 32 \quad \mathrm{x}^{\wedge} 9 \quad \mathrm{r}^{\wedge} 5\right)\right)-\mathrm{h} *(1) / 200 \quad(1 / 2033753534126478065664 \quad(-$ $1 / 203375353412647806566400(1+h) h^{\wedge} 13 r^{\wedge} 51-31 / 13240582904469258240000\left(1 / 32(1+h) h r^{\wedge} 5+1 / 32 h^{\wedge} 6 r^{\wedge} 5\right) h^{\wedge} 12 r^{\wedge} 46-$ $1540541 / 197726038040074256384000000 h^{\wedge} 18 r^{\wedge} 51+31 / 587202560000 h^{\wedge} 6 r^{\wedge} 21\left(-1 / 22548578304\left(1 / 32(1+h) h r^{\wedge} 5+1 / 32\right.\right.$

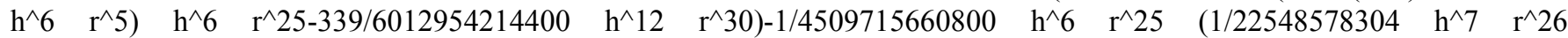

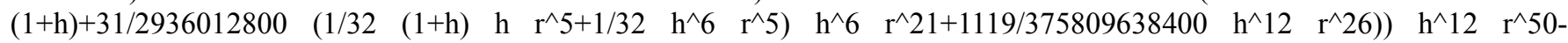
$31 / 132405829044692582400 \quad\left(1 / 219645381685659631091712 \quad\left(1 / 32 \quad(1+h) \quad \begin{array}{lllll}h & r^{\wedge} 5+1 / 32 & h^{\wedge} 6 & \left.r^{\wedge} 5\right) & h^{\wedge} 12\end{array}\right.\right.$ $\mathrm{r}^{\wedge} 50+1661509 / 17083529686662415751577600000 \mathrm{~h}^{\wedge} 18 \mathrm{r}^{\wedge} 55-1 / 4870492913664 \mathrm{~h}^{\wedge} 6 \mathrm{r}^{\wedge} 25(-1 / 22548578304(1 / 32(1+\mathrm{h}) \mathrm{h}$

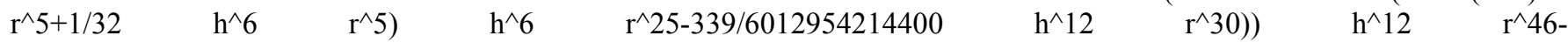
$15701517640283 / 165692679056158781069638940217915666371444736000000000 \quad h^{\wedge} 30$ $\mathrm{r}^{\wedge} 101+31 / 557305131435787132506149486592000 \mathrm{~h}^{\wedge} 18 \mathrm{r}^{\wedge} 71\left(-1 / 22548578304\left(1 / 32(1+\mathrm{h}) \mathrm{h} \mathrm{r}^{\wedge} 5+1 / 32 \mathrm{~h}^{\wedge} 6 \mathrm{r}^{\wedge} 5\right) \mathrm{h}^{\wedge} 6 \mathrm{r}^{\wedge} 25-\right.$ 
$\left.339 / 6012954214400 \quad h^{\wedge} 12 \quad \mathrm{r}^{\wedge} 30\right)-1 / 13574042241325137563395494695141376 \quad \mathrm{~h}^{\wedge} 18 \quad \mathrm{r}^{\wedge} 75 \quad\left(1 / 22548578304 \quad \mathrm{~h}^{\wedge} 7 \quad \mathrm{r}^{\wedge} 26\right.$ $\left.\left.(1+h)+31 / 2936012800\left(1 / 32(1+h) h r^{\wedge} 5+1 / 32 h^{\wedge} 6 r^{\wedge} 5\right) h^{\wedge} 6 r^{\wedge} 21+1119 / 375809638400 h^{\wedge} 12 r^{\wedge} 26\right)\right)$

$$
\underline{u}(x, \alpha)=\underline{u}_{0}(x, \alpha)+\sum_{m=1}^{2} \underline{u}_{m}(x, \alpha)
$$

$\underline{u}(x, \alpha)=$

$-\mathrm{h}^{*}\left(\mathrm{x}^{*} \mathrm{r}-(1 / 32) * \mathrm{x}^{\wedge} 9 * \mathrm{r}^{\wedge} 5\right)-(1+\mathrm{h}) * \mathrm{~h}^{*}\left(\mathrm{x}^{*} \mathrm{r}-(1 / 32) * \mathrm{x}^{\wedge}{ }^{*} \mathrm{r}^{\wedge} 5\right)-\mathrm{h}^{*}\left(-\mathrm{h}^{\wedge} 5^{*}(1 / 45097156608) * \mathrm{r}^{\wedge} 25^{*} \mathrm{x}^{\wedge} 49+(31 / 5872025600) * \mathrm{r}^{\wedge} 21 * \mathrm{x}^{\wedge} 41-\right.$ $\left.\left.(41 / 73400320) * \mathrm{r}^{\wedge} 17 * \mathrm{x}^{\wedge} 33+(3 / 81920) * \mathrm{r}^{\wedge} 13 * \mathrm{x}^{\wedge} 25-(3 / 2048) * \mathrm{r}^{\wedge} 9 * \mathrm{x}^{\wedge} 17+(1 / 32) * \mathrm{x}^{\wedge} 9 * \mathrm{r}^{\wedge} 5\right)\right)+(1+\mathrm{h}) *(-(1+\mathrm{h}) \quad \mathrm{h} \quad(\mathrm{x} \quad \mathrm{r}-1 / 32 \quad \mathrm{x} \wedge 9$ $\left.\mathrm{r}^{\wedge} 5\right)+\mathrm{h}^{\wedge} 6\left(-1 / 45097156608 \mathrm{r}^{\wedge} 25 \mathrm{x}^{\wedge} 49+31 / 5872025600 \mathrm{r}^{\wedge} 21 \mathrm{x}^{\wedge} 41-41 / 73400320 \mathrm{r}^{\wedge} 17 \mathrm{x}^{\wedge} 33+3 / 81920 \mathrm{r}^{\wedge} 13 \quad \mathrm{x}^{\wedge} 25-3 / 2048 \mathrm{r}^{\wedge} 9\right.$

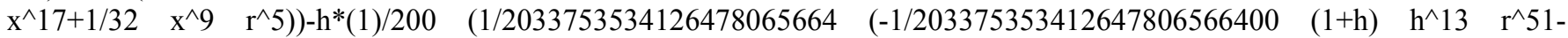
$31 / 13240582904469258240000\left(1 / 32(1+\mathrm{h}) \mathrm{h} \mathrm{r}^{\wedge} 5+1 / 32 \mathrm{~h}^{\wedge} 6 \mathrm{r}^{\wedge} 5\right) \mathrm{h}^{\wedge} 12 \mathrm{r}^{\wedge} 46-1540541 / 197726038040074256384000000 \mathrm{~h}^{\wedge} 18$ $\mathrm{r}^{\wedge} 51+31 / 587202560000 \mathrm{~h}^{\wedge} 6 \mathrm{r}^{\wedge} 21\left(-1 / 22548578304\left(1 / 32(1+\mathrm{h}) \mathrm{h} \mathrm{r}^{\wedge} 5+1 / 32 \mathrm{~h}^{\wedge} 6 \mathrm{r}^{\wedge} 5\right) \mathrm{h}^{\wedge} 6 \mathrm{r}^{\wedge} 25-339 / 6012954214400 \mathrm{~h}^{\wedge} 12 \mathrm{r}^{\wedge} 30\right)-$ $1 / 4509715660800 \quad h^{\wedge} 6 \quad r^{\wedge} 25 \quad\left(1 / 22548578304 \quad h^{\wedge} 7 \quad r^{\wedge} 26 \quad(1+h)+31 / 2936012800 \quad\left(1 / 32 \quad(1+h) \quad h \quad r^{\wedge} 5+1 / 32 \quad h^{\wedge} 6 \quad r^{\wedge} 5\right) \quad h^{\wedge} 6\right.$ $\left.\left.\mathrm{r}^{\wedge} 21+1119 / 375809638400 \mathrm{~h}^{\wedge} 12 \mathrm{r}^{\wedge} 26\right)\right) \mathrm{h}^{\wedge} 12 \mathrm{r}^{\wedge} 50-31 / 132405829044692582400(1 / 219645381685659631091712(1 / 32(1+\mathrm{h}) \mathrm{h}$ $\left.\mathrm{r}^{\wedge} 5+1 / 32 \quad \mathrm{~h}^{\wedge} 6 \quad \mathrm{r}^{\wedge} 5\right) \quad \mathrm{h}^{\wedge} 12 \quad \mathrm{r}^{\wedge} 50+1661509 / 17083529686662415751577600000 \quad \mathrm{~h}^{\wedge} 18 \quad \mathrm{r}^{\wedge} 55-1 / 4870492913664 \quad \mathrm{~h}^{\wedge} 6 \quad \mathrm{r}^{\wedge} 25 \quad(-$

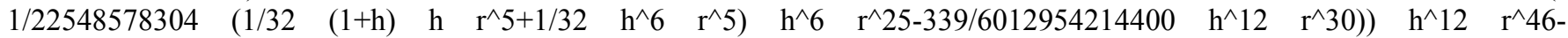
$15701517640283 / 165692679056158781069638940217915666371444736000000000$ $\mathrm{h}^{\wedge} 30$ $\mathrm{r}^{\wedge} 101+31 / 557305131435787132506149486592000 \mathrm{~h}^{\wedge} 18 \mathrm{r}^{\wedge} 71\left(-1 / 22548578304\left(1 / 32(1+\mathrm{h}) \mathrm{h} \mathrm{r}^{\wedge} 5+1 / 32 \mathrm{~h}^{\wedge} 6 \mathrm{r}^{\wedge} 5\right) \mathrm{h}^{\wedge} 6 \mathrm{r}^{\wedge} 25-\right.$ $\left.\left.339 / 6012954214400 \mathrm{~h}^{\wedge} 12 \mathrm{r}^{\wedge} 30\right)-1 / 13574042241325137563395494695141376 \mathrm{~h}^{\wedge} 18 \mathrm{r}^{\wedge} 75\right)$

$$
\begin{gathered}
\bar{u}_{0}(x, \alpha)=0 \\
\bar{u}_{1}(x, \alpha)=-\mathrm{h}^{*}\left(\mathrm{x} *(2-\mathrm{r})-(1 / 32) * \mathrm{x}^{\wedge} 9 *(2-\mathrm{r})^{\wedge} 5\right)
\end{gathered}
$$

$\bar{u}_{2}(x, \alpha)=-(1+\mathrm{h}) * \mathrm{~h} *\left(\mathrm{x} *(2-\mathrm{r})-(1 / 32)^{*} \mathrm{x}^{\wedge} 9^{*}(2-\mathrm{r})^{\wedge} 5\right)-\mathrm{h} *\left(-\mathrm{h}^{\wedge} 5^{*}\left(-(1 / 45097156608)^{*}(2-\mathrm{r})^{\wedge} 25^{*} \mathrm{x}^{\wedge} 49+(31 / 5872025600)^{*}(2-\right.\right.$ $\left.\left.r)^{\wedge} 21^{*} x^{\wedge} 41-(41 / 73400320)^{*}(2-r)^{\wedge} 17^{*} x^{\wedge} 33+(3 / 81920)^{*}(2-r)^{\wedge} 13^{*} x^{\wedge} 25-(3 / 2048)^{*}(2-r)^{\wedge} 9^{*} x^{\wedge} 17+(1 / 32)^{*} x^{\wedge} 9^{*}(2-r)^{\wedge} 5\right)\right)$

where

$$
\bar{R}_{0}(x, t, \alpha)=\left(x t\left(t, \overline{u_{0}}(t, \alpha)\right)^{3} \cdot G\left(t, \int_{a}^{t}\left(t, s, \bar{u}_{0}(s, \alpha)\right)^{2} d s\right)\right.
$$

$\bar{u}_{3}(x, \alpha)$

$=-h^{*}\left(\mathrm{x}^{*}(2-\mathrm{r})-(1 / 32)^{*} \mathrm{x}^{\wedge} 9 *(2-\mathrm{r})^{\wedge} 5\right)-(1+\mathrm{h}) * \mathrm{~h} *\left(\mathrm{x}^{*}(2-\mathrm{r})-(1 / 32)^{*} \mathrm{x}^{\wedge} 9 *(2-\mathrm{r})^{\wedge} 5\right)+\mathrm{h}^{\wedge} 6^{*}(-(1 / 45097156608) *(2-$

$\mathrm{r})^{\wedge} 25^{*} \mathrm{x}^{\wedge} 49+(31 / 5872025600)^{*}(2-\mathrm{r})^{\wedge} 21^{*} \mathrm{x}^{\wedge} 41-(41 / 73400320)^{*}(2-\mathrm{r})^{\wedge} 17^{*} \mathrm{x}^{\wedge} 33+(3 / 81920)^{*}(2-\mathrm{r})^{\wedge} 13^{*} \mathrm{x}^{\wedge} 25-(3 / 2048)^{*}(2-$

$\left.\mathrm{r})^{\wedge} 9^{*} \mathrm{x}^{\wedge} 17+(1 / 32)^{*} \mathrm{x}^{\wedge} 9 *(2-\mathrm{r})^{\wedge} 5\right)+(1+\mathrm{h})^{*}\left(-(1+\mathrm{h})^{*} \mathrm{~h}^{*}\left(\mathrm{x}^{*}(2-\mathrm{r})-(1 / 32)^{*} \mathrm{x}^{\wedge} 9^{*}(2-\mathrm{r})^{\wedge} 5\right)+\mathrm{h}^{\wedge} 6^{*}\left(-(1 / 45097156608)^{*}(2-\right.\right.$

$\mathrm{r})^{\wedge} 25^{*} \mathrm{x}^{\wedge} 49+(31 / 5872025600) *(2-\mathrm{r})^{\wedge} 21 * \mathrm{x}^{\wedge} 41-(41 / 73400320) *(2-\mathrm{r})^{\wedge} 17^{*} \mathrm{x}^{\wedge} 33+(3 / 81920)^{*}(2-\mathrm{r})^{\wedge} 13 * \mathrm{x}^{\wedge} 25-(3 / 2048) *(2-$

$\mathrm{r})^{\wedge} 9 * \mathrm{x}^{\wedge} 17+(1 / 32) * \mathrm{x}^{\wedge} 9 *(2 \mathrm{r}) \wedge 5 \mathrm{~h}^{*} \mathrm{x} *((1 / 208 *((1 / 2033753534126478065664 *((1 / 219645381685659631091712 *((1 / 32 *(1+\mathrm{h})) * \mathrm{~h}$ $\left.\left.*(2-r)^{\wedge} 5+(1 / 32)^{*} h^{\wedge} 6^{*}(2-r)^{\wedge} 5\right)\right)^{*} h^{\wedge} 12 *(2-r)^{\wedge} 50+(1661509 / 17083529686662415751577600000)^{*} h^{\wedge} 18 *(2-r)^{\wedge} 55-$

$(1 / 4870492913664) * h^{\wedge} 6 *(2-r)^{\wedge} 25^{*}\left(-\left(1 / 22548578304 *\left((1 / 32 *(1+h))^{*} h^{*}(2-r)^{\wedge} 5+(1 / 32)^{*} h^{\wedge} 6 *(2-r)^{\wedge} 5\right)\right)^{*} h^{\wedge} 6^{*}(2-r)^{\wedge} 25-\right.$

$\left.\left.\left.(339 / 6012954214400)^{*} h^{\wedge} 12^{*}(2 r)^{\wedge} 30\right)\right)\right)^{*} h^{\wedge} 12 *(2 r)^{\wedge} 50+(178007930970977 / 27836370081434675219699341956609831950402$ $7156480000000000)^{*} \mathrm{~h}^{\wedge} 30 *(2-\mathrm{r})^{\wedge} 105-(1 / 13574042241325137563395494695141376)^{*} \mathrm{~h}^{\wedge} 18^{*}(2-\mathrm{r})^{\wedge} 75^{*}(-$

$\left(1 / 22548578304 *\left((1 / 32 *(1+\mathrm{h}))^{*} \mathrm{~h}^{*}(2-\mathrm{r})^{\wedge} 5+(1 / 32)^{*} \mathrm{~h}^{\wedge} 6^{*}(2-\mathrm{r})^{\wedge} 5\right)\right)^{*} \mathrm{~h}^{\wedge} 6^{*}(2-\mathrm{r})^{\wedge} 25-(339 / 6012954214400)^{*} \mathrm{~h}^{\wedge} 12 *(2-$ $\left.\left.\left.\mathrm{r})^{\wedge} 30\right)\right)\right)^{*} \mathrm{x}^{\wedge} 209+\left(1 / 200 *\left(\left(1 / 2033753534126478065664 *\left(-(1 / 203375353412647806566400 *(1+\mathrm{h}))^{*} \mathrm{~h}^{\wedge} 13^{*}(2-\mathrm{r})^{\wedge} 51-\right.\right.\right.\right.$ $\left(31 / 13240582904469258240000 *\left((1 / 32 *(1+\mathrm{h}))^{*} \mathrm{~h}^{*}(2-\mathrm{r})^{\wedge} 5+(1 / 32)^{*} \mathrm{~h}^{\wedge} 6^{*}(2-\mathrm{r})^{\wedge} 5\right)\right)^{*} \mathrm{~h}^{\wedge} 12^{*}(2-\mathrm{r})^{\wedge} 46-$ $(1540541 / 197726038040074256384000000)^{*} h^{\wedge} 18^{*}(2-r)^{\wedge} 51+(31 / 587202560000)^{*} h^{\wedge} 6 *(2-r)^{\wedge} 21 *(-$ $\left.\left(1 / 22548578304 *\left((1 / 32 *(1+\mathrm{h}))^{*} \mathrm{~h}^{*}(2-\mathrm{r})^{\wedge} 5+(1 / 32)^{*} \mathrm{~h}^{\wedge} 6 *(2-\mathrm{r})^{\wedge} 5\right)\right)^{*} \mathrm{~h}^{\wedge} 6^{*}(2-\mathrm{r})^{\wedge} 25-(339 / 6012954214400)^{*} \mathrm{~h}^{\wedge} 12^{*}(2-\mathrm{r})^{\wedge} 30\right)-$ $(1 / 4509715660800)^{*} \mathrm{~h}^{\wedge} 6^{*}(2-\mathrm{r})^{\wedge} 25^{*}\left((1 / 22548578304)^{*} \mathrm{~h}^{\wedge} 7^{*}(2-\mathrm{r})^{\wedge} 26^{*}(1+\mathrm{h})+\left(31 / 2936012800 *\left((1 / 32 *(1+\mathrm{h}))^{*} \mathrm{~h}^{*}(2-\right.\right.\right.$ r $\left.\left.\left.\left.\left.)^{\wedge} 5+(1 / 32)^{*} h^{\wedge} 6^{*}(2-r)^{\wedge} 5\right)\right)^{*} h^{\wedge} 6^{*}(2-r)^{\wedge} 21+(1119 / 375809638400) * h^{\wedge} 12 *(2-r)^{\wedge} 26\right)\right)\right)^{*} h^{\wedge} 12^{*}(2-r)^{\wedge} 50-$

$\left(31 / 132405829044692582400 *\left(\left(1 / 219645381685659631091712 *\left((1 / 32 *(1+\mathrm{h}))^{*} \mathrm{~h}^{*}(2-\mathrm{r})^{\wedge} 5+(1 / 32)^{*} \mathrm{~h}^{\wedge} 6^{*}(2-\mathrm{r})^{\wedge} 5\right)\right)^{*} \mathrm{~h}^{\wedge} 12 *(2-\right.\right.$ $\mathrm{r})^{\wedge} 50+(1661509 / 17083529686662415751577600000)^{*} \mathrm{~h}^{\wedge} 18 *(2-\mathrm{r})^{\wedge} 55-(1 / 4870492913664)^{*} \mathrm{~h}^{\wedge} 6 *(2-\mathrm{r})^{\wedge} 25^{*}(-$ $\left(1 / 22548578304 *\left((1 / 32 *(1+\mathrm{h}))^{*} \mathrm{~h}^{*}(2-\mathrm{r})^{\wedge} 5+(1 / 32)^{*} \mathrm{~h}^{\wedge} 6^{*}(2-\mathrm{r})^{\wedge} 5\right)\right)^{*} \mathrm{~h}^{\wedge} 6^{*}(2-\mathrm{r})^{\wedge} 25-(339 / 6012954214400)^{*} \mathrm{~h}^{\wedge} 12 *(2-$ $\left.\left.\left.\mathrm{r})^{\wedge} 30\right)\right)\right)^{*} \mathrm{~h}^{\wedge} 12 *(2-\mathrm{r})^{\wedge} 46-(15701517640283 / 165692679056158781069638940217915666371444736000000000) * \mathrm{~h}^{\wedge} 30^{*}(2-$ $\mathrm{r})^{\wedge} 101+(31 / 557305131435787132506149486592000) * h^{\wedge} 18^{*}(2-\mathrm{r})^{\wedge} 71 *\left(-\left(1 / 22548578304 *\left((1 / 32 *(1+\mathrm{h}))^{*} \mathrm{~h} *(2-\right.\right.\right.$ $\left.\left.r)^{\wedge} 5+(1 / 32)^{*} h^{\wedge} 6^{*}(2-r)^{\wedge} 5\right)\right)^{*} h^{\wedge} 6^{*}(2-r)^{\wedge} 25-(339 / 6012954214400)^{*} h^{\wedge} 12^{*}(2-r)^{\wedge} 30$

$$
\bar{u}(x, \alpha)=\bar{u}_{0}(x, \alpha)+\sum_{m=1}^{2} \bar{u}_{m}(x, \alpha)
$$


$\bar{u}(x, \alpha)=$

$-\mathrm{h}\left(\mathrm{x}^{*}(2-\mathrm{r})-(1 / 32)^{*} \mathrm{x}^{\wedge} 9 *(2-\mathrm{r})^{\wedge} 5\right)-(1+\mathrm{h}) * \mathrm{~h} *\left(\mathrm{x}^{*}(2-\mathrm{r})-(1 / 32)^{*} \mathrm{x}^{\wedge} 9^{*}(2-\mathrm{r})^{\wedge} 5\right)-\mathrm{h} *\left(-\mathrm{h}^{\wedge} 5 *(-(1 / 45097156608) *(2-\right.$

$\mathrm{r})^{\wedge} 25^{*} \mathrm{x}^{\wedge} 49+(31 / 5872025600) *(2-\mathrm{r})^{\wedge} 21^{*} \mathrm{x}^{\wedge} 41-(41 / 73400320)^{*}(2-\mathrm{r})^{\wedge} 17^{*} \mathrm{x}^{\wedge} 33+(3 / 81920)^{*}(2-\mathrm{r})^{\wedge} 13 * \mathrm{x}^{\wedge} 25-(3 / 2048) *(2-$

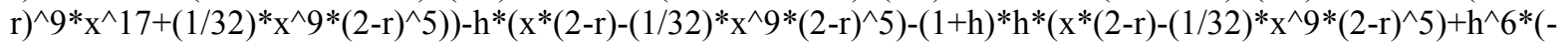

$(1 / 45097156608)^{*}(2-r)^{\wedge} 25^{*} \mathrm{x}^{\wedge} 49+(31 / 5872025600) *(2-\mathrm{r})^{\wedge} 21^{*} \mathrm{x}^{\wedge} 41-(41 / 73400320)^{*}(2-\mathrm{r})^{\wedge} 17^{*} \mathrm{x}^{\wedge} 33+(3 / 81920)^{*}(2-\mathrm{r})^{\wedge} 13^{*} \mathrm{x}^{\wedge} 25-$

$\left.(3 / 2048)^{*}(2-\mathrm{r})^{\wedge} 9^{*} \mathrm{x}^{\wedge} 17+(1 / 32)^{*} \mathrm{x}^{\wedge} 9^{*}(2-\mathrm{r})^{\wedge} 5\right)+(1+\mathrm{h})^{*}\left(-(1+\mathrm{h}) * \mathrm{~h} *\left(\mathrm{x}^{*}(2-\mathrm{r})-(1 / 32)^{*} \mathrm{x}^{\wedge} 9^{*}(2-\mathrm{r})^{\wedge} 5\right)+\mathrm{h}^{\wedge} 6^{*}\left(-(1 / 45097156608)^{*}(2\right.\right.$

r) $25 * x^{\wedge} 49+(31 / 5872025600)^{*}(2-r)^{\wedge} 21^{*} \mathrm{x}^{\wedge} 41-(41 / 73400320)^{*}(2-\mathrm{r})^{\wedge} 17^{*} \mathrm{x}^{\wedge} 33+(3 / 81920) *(2-\mathrm{r})^{\wedge} 13 * \mathrm{x}^{\wedge} 25-(3 / 2048)^{*}(2-$

$\left.\left.\mathrm{r})^{\wedge} 9^{*} \mathrm{x}^{\wedge} 17+(1 / 32)^{*} \mathrm{x}^{\wedge} 9^{*}(2 \mathrm{r})^{\wedge} 5\right)\right) \mathrm{h} * \mathrm{x}^{*}((1 / 208 *((1 / 2033753534126478065664 *((1 / 219645381685659631091712 *((1 / 32 *(1+\mathrm{h}))$

$\left.\left.* \mathrm{~h}^{*}(2-\mathrm{r})^{\wedge} 5+(1 / 32)^{*} \mathrm{~h}^{\wedge} 6^{*}(2-\mathrm{r})^{\wedge} 5\right)\right)^{*} \mathrm{~h}^{\wedge} 12^{*}(2-\mathrm{r})^{\wedge} 50+(1661509 / 17083529686662415751577600000) * \mathrm{~h}^{\wedge} 18^{*}(2-\mathrm{r})^{\wedge} 55-$

$(1 / 4870492913664)^{*} \mathrm{~h}^{\wedge} 6^{*}(2-\mathrm{r})^{\wedge} 25^{*}\left(-\left(1 / 22548578304 *\left((1 / 32 *(1+\mathrm{h}))^{*} \mathrm{~h} *(2-\mathrm{r})^{\wedge} 5+(1 / 32)^{*} \mathrm{~h}^{\wedge} 6^{*}(2-\mathrm{r})^{\wedge} 5\right)\right)^{*} \mathrm{~h}^{\wedge} 6^{*}(2-\mathrm{r})^{\wedge} 25-\right.$

$\left.\left.\left.(339 / 6012954214400)^{*} \mathrm{~h}^{\wedge} 12^{*}(2 \mathrm{r})^{\wedge} 30\right)\right)\right)^{*} \mathrm{~h}^{\wedge} 12^{*}(2 \mathrm{r})^{\wedge} 50+(178007930970977 / 27836370081434675219699341956609831950402$ $7156480000000000)^{*} \mathrm{~h}^{\wedge} 30^{*}(2-\mathrm{r})^{\wedge} 105-(1 / 13574042241325137563395494695141376) * \mathrm{~h}^{\wedge} 18^{*}(2-\mathrm{r})^{\wedge} 75^{*}(-$

$\left(1 / 22548578304 *\left((1 / 32 *(1+\mathrm{h}))^{*} \mathrm{~h}^{*}(2-\mathrm{r})^{\wedge} 5+(1 / 32)^{*} \mathrm{~h}^{\wedge} 6^{*}(2-\mathrm{r})^{\wedge} 5\right)\right)^{*} \mathrm{~h}^{\wedge} 6^{*}(2-\mathrm{r})^{\wedge} 25-(339 / 6012954214400)^{*} \mathrm{~h}^{\wedge} 12 *(2-$

r)^30)) $)^{\wedge} \mathrm{x}^{\wedge} 209+\left(1 / 200 *\left(\left(1 / 2033753534126478065664 *\left(-(1 / 203375353412647806566400 *(1+\mathrm{h}))^{*} \mathrm{~h}^{\wedge} 13 *(2-\mathrm{r})^{\wedge} 51-\right.\right.\right.\right.$

$\left(31 / 13240582904469258240000 *\left((1 / 32 *(1+\mathrm{h}))^{*} \mathrm{~h}^{*}(2-\mathrm{r})^{\wedge} 5+(1 / 32)^{*} \mathrm{~h}^{\wedge} 6^{*}(2-\mathrm{r})^{\wedge} 5\right)\right)^{*} \mathrm{~h}^{\wedge} 12 *(2-\mathrm{r})^{\wedge} 46-$

$(1540541 / 197726038040074256384000000)^{*} \mathrm{~h}^{\wedge} 18^{*}(2-\mathrm{r})^{\wedge} 51+(31 / 587202560000)^{*} \mathrm{~h}^{\wedge} 6 *(2-\mathrm{r})^{\wedge} 21 *(-$

$\left.\left(1 / 22548578304 *\left((1 / 32 *(1+\mathrm{h}))^{*} \mathrm{~h} *(2-\mathrm{r})^{\wedge} 5+(1 / 32)^{*} \mathrm{~h}^{\wedge} 6^{*}(2-\mathrm{r})^{\wedge} 5\right)\right)^{*} \mathrm{~h}^{\wedge} 6^{*}(2-\mathrm{r})^{\wedge} 25-(339 / 6012954214400)^{*} \mathrm{~h}^{\wedge} 12 *(2-\mathrm{r})^{\wedge} 30\right)-$

$(1 / 4509715660800)^{*} \mathrm{~h}^{\wedge} 6 *(2-\mathrm{r})^{\wedge} 25^{*}\left((1 / 22548578304)^{*} \mathrm{~h}^{\wedge} 7 *(2-\mathrm{r})^{\wedge} 26^{*}(1+\mathrm{h})+\left(31 / 2936012800 *\left((1 / 32 *(1+\mathrm{h}))^{*} \mathrm{~h} *(2-\right.\right.\right.$

r) $\left.\left.\left.\left.\left.{ }^{\wedge} 5+(1 / 32)^{*} \mathrm{~h}^{\wedge} 6^{*}(2-\mathrm{r})^{\wedge} 5\right)\right)^{*} \mathrm{~h}^{\wedge} 6^{*}(2-\mathrm{r})^{\wedge} 21+(1119 / 375809638400)^{*} \mathrm{~h}^{\wedge} 12^{*}(2-\mathrm{r})^{\wedge} 26\right)\right)\right)^{*} \mathrm{~h}^{\wedge} 12 *(2-\mathrm{r})^{\wedge} 50-$

$\left(31 / 132405829044692582400 *\left(\left(1 / 219645381685659631091712 *\left((1 / 32 *(1+\mathrm{h}))^{*} \mathrm{~h} *(2-\mathrm{r})^{\wedge} 5+(1 / 32)^{*} \mathrm{~h}^{\wedge} 6 *(2-\mathrm{r})^{\wedge} 5\right)\right)^{*} \mathrm{~h}^{\wedge} 12^{*}(2-\right.\right.$

r)^50+(1661509/17083529686662415751577600000)*h^18*(2-r)^55-(1/4870492913664)* $\mathrm{h}^{\wedge} 6 *(2-\mathrm{r})^{\wedge} 25^{*}(-$

$\left(1 / 22548578304 *\left((1 / 32 *(1+\mathrm{h}))^{*} \mathrm{~h} *(2-\mathrm{r})^{\wedge} 5+(1 / 32)^{*} \mathrm{~h}^{\wedge} 6^{*}(2-\mathrm{r})^{\wedge} 5\right)\right)^{*} \mathrm{~h}^{\wedge} 6 *(2-\mathrm{r})^{\wedge} 25-(339 / 6012954214400) * \mathrm{~h}^{\wedge} 12 *(2-$

r)^30)) $)^{*} h^{\wedge} 12 *(2-r)^{\wedge} 46$

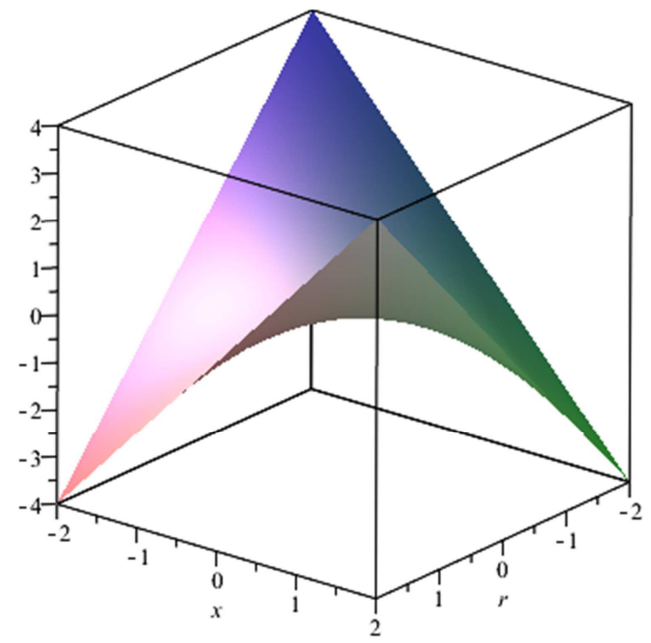

Figure 1a. The exact solution $\underline{\boldsymbol{u}}(\boldsymbol{x}, \boldsymbol{\alpha})$.

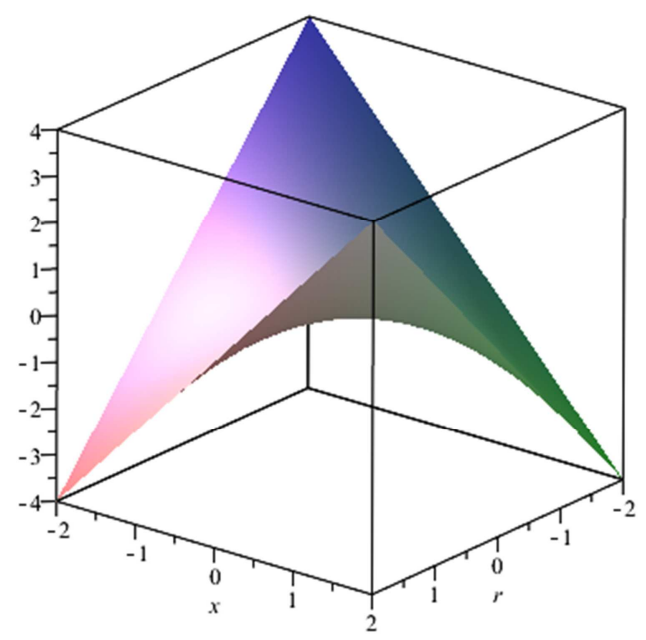

Figure 1b. The approximate solution $\underline{\boldsymbol{u}}(\boldsymbol{x}, \boldsymbol{\alpha})$.

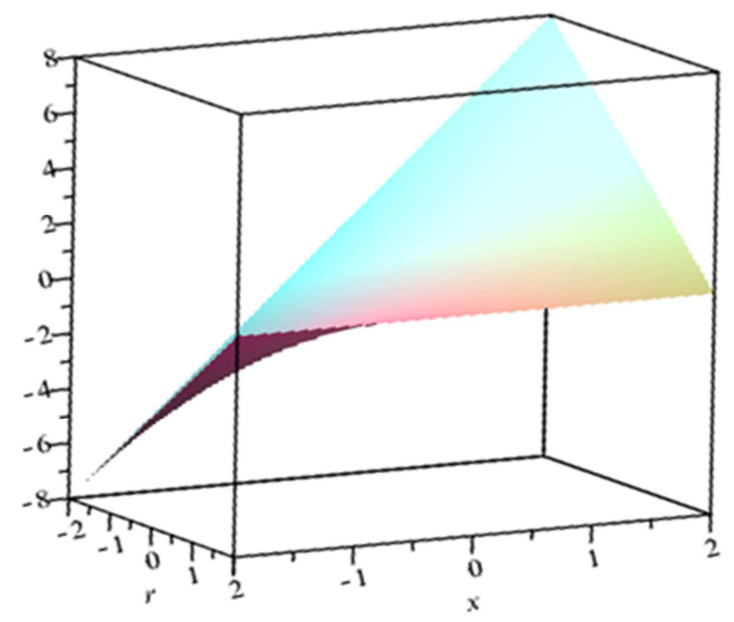

Figure 2a. The exact solution $\overline{\boldsymbol{u}}(\boldsymbol{x}, \boldsymbol{\alpha})$.

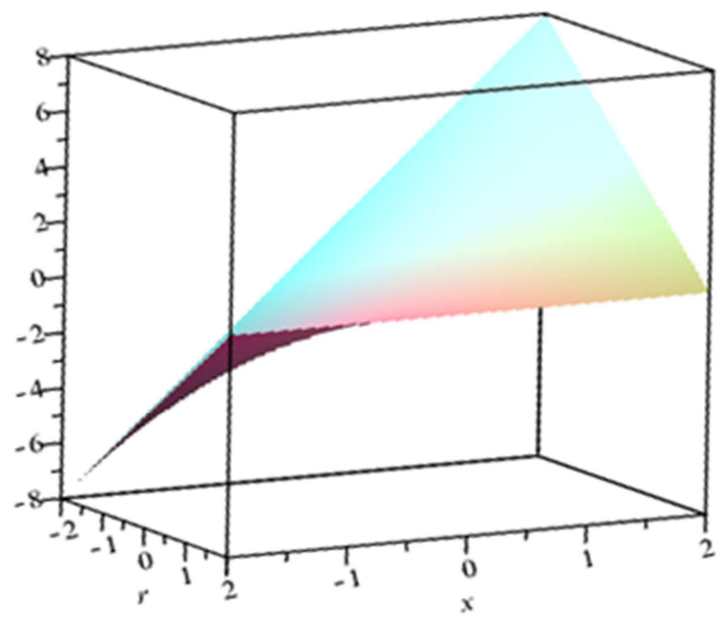

Figure $2 \boldsymbol{b}$. The approximate solution $\overline{\boldsymbol{u}}(\boldsymbol{x}, \boldsymbol{\alpha})$. 
as long as a series solution given by the HAM converges, it must be the exact solution. So it is important to ensure that the homotopy solution series converges. contains the auxiliary parameter $\hbar$, which provides us a simple way to adjust and control the convergence of the solution series. In general, by means of the so-called $\hbar$-curve (a curve of a versus $\hbar)$, it is straightforward to choose an appropriate range for $\hbar$ which ensures the convergence of the solution series. As pointed by Liao [13], the valid region of $\hbar$ is a horizontal line segment. In Fig. (1a), Fig(1b) and Fig(2a), $\operatorname{Fig}(2 \mathrm{~b})$ we plot the $\hbar$-curves, we could find that if $\hbar$ is about in area $[-1,-0.7]$ the result is convergent. In table (1) we introduce the error between the exact and approximate solutions of HAM for different values of $\hbar$

Table 1. Computation between the exact and HAM $h=-1$ in case 1 and determine the absolute error.

\begin{tabular}{|c|c|c|c|c|c|c|}
\hline$x$ & $\operatorname{exact} \underline{u}(x, r)$ & $\begin{array}{l}H A M \underline{u}(x, r) \\
\alpha=0.1\end{array}$ & $\operatorname{exact} \underline{u}(x, r)$ & $\begin{array}{l}H A M \underline{u}(x, r) \\
\alpha=0.5\end{array}$ & exact $\underline{u}(x, r)$ & $\begin{array}{l}H A M \underline{u}(x, r) \\
\alpha=1\end{array}$ \\
\hline 0 & 0.0000000 & 0.00000 & 0.000000 & 0.00000 & 0.00000 & 0.00000 \\
\hline 0.2 & 0.0200000 & 0.02000 & 0.100000 & 0.10000 & 0.20000 & 0.20000 \\
\hline 0.6 & 0.0600000 & 0.06000 & 0.300000 & 0.29999 & 0.60000 & 0.59999 \\
\hline 0.8 & 0.0800000 & 0.08000 & 0.400000 & 0.39999 & 0.80000 & 0.79999 \\
\hline 1 & 0.100000 & 0.10000 & 0.500000 & 0.49999 & 1.00000 & 0.99999 \\
\hline$x$ & $\operatorname{exact} \bar{u}(x, r)$ & $\begin{array}{l}H A M \bar{u}(x, r) \\
\alpha=0.1\end{array}$ & exact $\bar{u}(x, r)$ & $\begin{array}{l}H A M \bar{u}(x, r) \\
\alpha=0.5\end{array}$ & $\operatorname{exact} \bar{u}(x, r)$ & $\begin{array}{l}H A M \bar{u}(x, r) \\
\alpha=1\end{array}$ \\
\hline 0 & 0.00000 & 0.00000 & 0.00000 & 0.00000 & 0.00000 & 0.00000 \\
\hline 0.2 & 0.38000 & 0.38000 & 0.30000 & 0.30000 & 0.20000 & 0.20000 \\
\hline 0.4 & 0.76000 & 0.75999 & 0.60000 & 0.59999 & 0.40000 & 0.39999 \\
\hline 0.6 & 1.14000 & 1.13992 & 0.90000 & 0.89991 & 0.60000 & 0.59999 \\
\hline 0.8 & 1.52000 & 1.51992 & 1.20000 & 1.19876 & 0.80000 & 0.79999 \\
\hline 1 & 1.90000 & 1.87556 & 1.50000 & 1.45029 & 1.00000 & 0.99999 \\
\hline
\end{tabular}

Example 2:

Now we discuss this example by using HAM

$$
\begin{gathered}
\overline{k\left(x, t, F_{1 \alpha}(x, t, u(t, \alpha))\right) \cdot G\left(t, \int_{a}^{t} F_{2 \alpha}(t, s, u(s, r)) d s\right)}<\frac{0, k\left(x, t, F_{1 \alpha}(x, t, u(t, \alpha))\right) \cdot G\left(t, \int_{a}^{t} F_{2 \alpha}(t, s, u(s, r)) d s\right)<0}{\underline{u}(x, \alpha)=} \underline{\underline{f}(x, \alpha)+\lambda \int_{a}^{x} k\left(x, t, \bar{F}_{1 \alpha}(x, t, \bar{u}(t, \alpha))\right) \cdot G\left(t, \int_{a}^{t} \bar{F}_{2 \alpha}(t, s, \bar{u}(s, r)) d s\right) d t} \\
\bar{u}(x, \alpha)=\bar{f}(x, \alpha)+\lambda \int_{a}^{x} k\left(x, t, \underline{F}_{1 \alpha}(x, t, \underline{u}(t, \alpha))\right) \cdot G\left(t, \int_{a}^{t} \underline{F}_{2 \alpha}(t, s, \underline{u}(s, r)) d s\right) d t
\end{gathered}
$$

Where

$$
\begin{gathered}
\underline{F}_{1 \alpha}(x, u(x, \alpha))=(\underline{u}(x, \alpha))^{2} \\
\underline{F}_{2 \alpha}(x, u(x, \alpha))=(\underline{u}(x, \alpha))^{3} \\
\bar{F}_{1 \alpha}(x, u(x, \alpha))=(\bar{u}(x, \alpha))^{2} \\
\bar{F}_{2 \alpha}(x, u(x, \alpha))=(\bar{u}(x, \alpha))^{3} \\
k\left(x, t, \bar{F}_{1 \alpha}(t, \bar{u}(t, \alpha)) \cdot G\left(t, \int_{a}^{t} \bar{F}_{2 \alpha}(t, s, \bar{u}(s, \alpha)) d s\right)=\left(x t(t, \bar{u}(t, \alpha))^{3}, \int_{a}^{t}(s, \bar{u}(s, \alpha))^{2} d s\right)\right. \\
k\left(x, t, \underline{F}_{1 \alpha}(t, \underline{u}(t, \alpha))\right) \cdot G\left(t, \int_{a}^{t} \underline{F}_{2 \alpha}(t, s, \underline{u}(s, \alpha)) d s\right)=\left(x t(t, \underline{u}(t, \alpha))^{2}, \int_{a}^{t}(t, s, \underline{u}(s, \alpha))^{3} d s\right)
\end{gathered}
$$

with the exact solution $\underline{u}(x, \alpha)=x \alpha$ and $\bar{u}(x, \alpha)=x(2-\alpha)$

the intimal condition is $\underline{u}_{0}(x, \alpha)=\bar{u}_{0}(x, \alpha)=0$, and $0 \leq \alpha \leq 1$

$$
\underline{f}(x, r)=x * r-(1 / 32) * x^{\wedge} 9 *(2-r)^{\wedge} 5
$$




$$
\bar{f}(x, r)=x *(2-r)-(1 / 32) * x^{\wedge} 9 * r^{\wedge} 5
$$

By using HAM to solve the system above

$$
\begin{gathered}
\underline{u}_{0}(x, \alpha)=0 \\
\underline{u}_{1}(x, \alpha)=-\mathrm{h}^{*}\left(\mathrm{x}^{*} \mathrm{r}-(1 / 32)^{*} \mathrm{x}^{\wedge} 9^{*}(2-\mathrm{r})^{\wedge} 5\right) \\
\underline{u}_{2}(x, \alpha)=
\end{gathered}
$$

$-(1+\mathrm{h}) * \mathrm{~h} *\left(\mathrm{x}^{*} \mathrm{r}-(1 / 32) * \mathrm{x}^{\wedge} 9 *(2-\mathrm{r})^{\wedge} 5\right)-\mathrm{h}^{*}\left(-\mathrm{h}^{\wedge} 5^{*}\left(-(1 / 45097156608) * \mathrm{r}^{\wedge} 25^{*} \mathrm{x}^{\wedge} 49+(31 / 5872025600 *(2-\mathrm{r}))^{*} \mathrm{r}^{\wedge} 20 * \mathrm{x}^{\wedge} 41-\right.\right.$ $\left.\left.(41 / 73400320) *(2-r)^{\wedge} 2^{*} \mathrm{r}^{\wedge} 15^{*} \mathrm{x}^{\wedge} 33+(3 / 81920)^{*}(2-\mathrm{r})^{\wedge} 3 * \mathrm{r}^{\wedge} 10^{*} \mathrm{x}^{\wedge} 25-(3 / 2048)^{*}(2-\mathrm{r})^{\wedge} 4^{*} \mathrm{r}^{\wedge} 5^{*} \mathrm{x}^{\wedge} 17+(1 / 32)^{*} \mathrm{x}^{\wedge} 9^{*}(2-\mathrm{r})^{\wedge} 5\right)\right)$

$$
\underline{u}_{3}(x, \alpha)=
$$

$(1+\mathrm{h}) *\left(-(1+\mathrm{h}) * \mathrm{~h} *\left(\mathrm{x}^{*} \mathrm{r}-(1 / 32) * \mathrm{x}^{\wedge} 9 *(2-\mathrm{r})^{\wedge} 5\right)+\mathrm{h}^{\wedge} 6^{*}\left(-(1 / 45097156608) * \mathrm{r}^{\wedge} 25 * \mathrm{x}^{\wedge} 49+(31 / 5872025600 *(2-\mathrm{r}))^{*} \mathrm{r}^{\wedge} 20 * \mathrm{x}^{\wedge} 41-\right.\right.$ $\left.\left.(41 / 73400320)^{*}(2-r)^{\wedge} 2 * r^{\wedge} 15 * x^{\wedge} 33+(3 / 81920)^{*}(2-r)^{\wedge} 3 * r^{\wedge} 10^{*} x^{\wedge} 25-(3 / 2048)^{*}(2-r)^{\wedge} 4 * r^{\wedge} 5 * x^{\wedge} 17+(1 / 32)^{*} x^{\wedge} 9^{*}(2-r)^{\wedge} 5\right)\right)-$ $\mathrm{h}^{*} \mathrm{x}^{*}\left(\left(1 / 40^{*}\left((1 / 4)^{*}(1+\mathrm{h})^{\wedge} 3 * \mathrm{~h}^{\wedge} 3 *(2 \mathrm{r})^{\wedge} 3 *\left((41 / 36700160 *(1+\mathrm{h}))^{*} \mathrm{~h}^{\wedge} 7 *(2 \mathrm{r})^{\wedge} 16^{*} \mathrm{r}^{\wedge} 2+\left(3 / 40960 *\left((1 / 32 *(1+\mathrm{h}))^{*} \mathrm{~h}^{*} \mathrm{r}^{\wedge} 5+(1 / 32)^{*} \mathrm{~h}^{\wedge} 6\right.\right.\right.\right.\right.\right.$ $\left.\left.\left.{ }^{*} \mathrm{r}^{\wedge} 5\right)\right)^{*} \mathrm{~h}^{\wedge} 6 * \mathrm{r}^{\wedge} 3 *(2 \mathrm{r})^{\wedge} 10+(9 / 4194304)^{*} \mathrm{~h}^{\wedge} 12 * \mathrm{r}^{\wedge} 8 *(2 \mathrm{r})^{\wedge} 10\right)+(1 / 4)^{*}(1+\mathrm{h})^{\wedge} 2 * \mathrm{~h}^{\wedge} 2^{*}(2 \mathrm{r})^{\wedge} 2^{*}\left(\left(1 / 32^{*}(1+\mathrm{h})\right)^{*} \mathrm{~h}^{*} \mathrm{r}^{\wedge} 5+(1 / 32)^{*} \mathrm{~h}^{\wedge} 6 * \mathrm{r}^{\wedge} 5\right)^{*}$ $\left(-(3 / 40960 *(1+\mathrm{h}))^{*} \mathrm{~h}^{\wedge} 7 *(2-\mathrm{r})^{\wedge} 11 * \mathrm{r}^{\wedge} 3-\left(3 / 1024 *\left((1 / 32 *(1+\mathrm{h}))^{*} \mathrm{~h}^{*} \mathrm{r}^{\wedge} 5+(1 / 32)^{*} \mathrm{~h}^{\wedge} 6 * \mathrm{r}^{\wedge} 5\right)\right)^{*} \mathrm{~h}^{\wedge} 6 * \mathrm{r}^{\wedge} 4 *(2-\mathrm{r})^{\wedge} 5\right)+\left(\left(1 / 20^{*}(1+\mathrm{h})\right)^{*} \mathrm{~h}^{*}(2-\right.$ $\mathrm{r})^{*}\left((3 / 1024 *(1+\mathrm{h}))^{*} \mathrm{~h}^{\wedge} 7 *(2 \mathrm{r})^{\wedge} 6^{*} \mathrm{r}^{\wedge} 4+\left((1 / 32 *(1+\mathrm{h}))^{*} \mathrm{~h}^{*} \mathrm{r}^{\wedge} 5+(1 / 32)^{*} \mathrm{~h}^{\wedge} 6^{*} \mathrm{r}^{\wedge} 5\right)^{\wedge} 2\right)(1 / 10)^{*}\left(\left(1 / 32^{*}(1+\mathrm{h})\right)^{*} \mathrm{~h}^{*} \mathrm{r}^{\wedge} 5+(1 / 32)^{*} \mathrm{~h}^{\wedge} 6^{*} \mathrm{r}^{\wedge} 5\right)^{\wedge}$ $\left.2 *(1+\mathrm{h}) * h^{*}(2 \mathrm{r})(3 / 40960) * \mathrm{~h}^{\wedge} 8 * \mathrm{r}^{\wedge} 4 *(2 \mathrm{r})^{\wedge} 7 *(1+\mathrm{h})^{\wedge} 2\right) *\left((3 / 1024 *(1+\mathrm{h}))^{*} \mathrm{~h}^{\wedge} 7 *(2 \mathrm{r})^{\wedge} 6^{*} \mathrm{r}^{\wedge} 4+\left((1 / 32 *(1+\mathrm{h}))^{*} \mathrm{~h}^{*} \mathrm{r}^{\wedge} 5+(1 / 32)^{*} \mathrm{~h}^{\wedge} 6^{*} \mathrm{r}^{\wedge} 5\right)\right.$

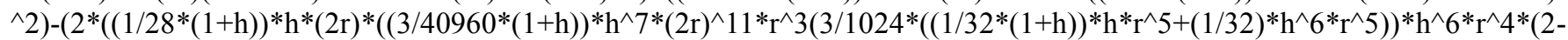
$\left.\mathrm{r})^{\wedge} 5\right)+\left(1 / 28 *\left((1 / 32 *(1+\mathrm{h}))^{*} \mathrm{~h}^{*} \mathrm{r}^{\wedge} 5+(1 / 32)^{*} \mathrm{~h}^{\wedge} 6^{*} \mathrm{r}^{\wedge} 5\right)\right) *\left((3 / 1024 *(1+\mathrm{h}))^{*} \mathrm{~h}^{\wedge} 7 *(2 \mathrm{r})^{\wedge} 6^{*} \mathrm{r}^{\wedge} 4+\left((1 / 32 *(1+\mathrm{h}))^{*} \mathrm{~h}^{*} \mathrm{r}^{\wedge} 5+(1 / 32)^{*} \mathrm{~h}^{\wedge} 6 * \mathrm{r}^{\wedge} 5\right)\right.$ $\left.\left.\left.{ }^{\wedge}\right)+(3 / 28672)^{*} h^{\wedge} 7 * r^{\wedge} 4 *(2 r)^{\wedge} 6^{*}(1+h)^{*}\left(\left(1 / 32^{*}(1+h)\right)^{*} h^{*} r^{\wedge} 5+(1 / 32)^{*} h^{\wedge} 6^{*} r^{\wedge} 5\right)+(3 / 2293760)^{*} h^{\wedge} 8^{*} r^{\wedge} 3 *(2 r)^{\wedge} 12^{*}(1+h)^{\wedge} 2\right)\right)^{*}(1+h$ )$^{*} h^{*}(2 \mathrm{r}) *\left((1 / 32 *(1+\mathrm{h}))^{*} \mathrm{~h}^{*} \mathrm{r}^{\wedge} 5+(1 / 32) * \mathrm{~h}^{\wedge} 6^{*} \mathrm{r}^{\wedge} 5\right)+\left((1 / 36 *(1+\mathrm{h}))^{*} \mathrm{~h} *(2 \mathrm{r}) *\left((41 / 36700160 *(1+\mathrm{h}))^{*} \mathrm{~h}^{\wedge} 7 *(2 \mathrm{r})^{\wedge} 16^{*} \mathrm{r}^{\wedge} 2+\left(3 / 40960^{*}((1\right.\right.\right.$ $\left.\left.\left./ 32 *(1+h))^{*} h^{*} r^{\wedge} 5+(1 / 32)^{*} h^{\wedge} 6 * r^{\wedge} 5\right)\right)^{*} h^{\wedge} 6 * r^{\wedge} 3 *(2 r)^{\wedge} 10+(9 / 4194304) * h^{\wedge} 12 * r^{\wedge} 8 *(2 r)^{\wedge} 10\right)+\left(1 / 36 *\left((1 / 32 *(1+h))^{*} h^{*} r^{\wedge} 5+(1 / 32) * h\right.\right.$ $\left.\left.{ }^{\wedge} 6^{*} \mathrm{r}^{\wedge} 5\right)\right)^{*}\left(-(3 / 40960 *(1+\mathrm{h}))^{*} \mathrm{~h}^{\wedge} 7 *(2-\mathrm{r})^{\wedge} 11 * \mathrm{r}^{\wedge} 3-\left(3 / 1024 *\left((1 / 32 *(1+\mathrm{h}))^{*} \mathrm{~h}^{*} \mathrm{r}^{\wedge} 5+(1 / 32)^{*} \mathrm{~h}^{\wedge} 6^{*} \mathrm{r}^{\wedge} 5\right)\right)^{*} \mathrm{~h}^{\wedge} 6^{*} \mathrm{r}^{\wedge} 4 *(2-\mathrm{r})^{\wedge} 5\right)-$ $(1 / 24576) * h^{\wedge} 6 * r^{\wedge} 4 *(2-r)^{\wedge} 5 *\left((3 / 1024 *(1+h))^{*} h^{\wedge} 7 *(2-r)^{\wedge} 6 * r^{\wedge} 4+\left((1 / 32 *(1+h))^{*} h^{*} \mathrm{r}^{\wedge} 5+(1 / 32)^{*} h^{\wedge} 6 * r^{\wedge} 5\right)^{\wedge} 2\right)-$ $(1 / 491520) * h^{\wedge} 7 * r^{\wedge} 3 *(2-r) \wedge 11 *(1+h) *\left((1 / 32 *(1+h)) * h^{*} r^{\wedge} 5+(1 / 32) * h^{\wedge} 6 * r^{\wedge} 5\right.$

$$
\begin{gathered}
\underline{u}(x, \alpha)=\underline{u}_{0}(x, \alpha)+\sum_{m=1}^{2} \underline{u}_{m}(x, \alpha) \\
\underline{u}(x, \alpha)=
\end{gathered}
$$

$-\mathrm{h}^{*}\left(\mathrm{x}^{*} \mathrm{r}-(1 / 32) * \mathrm{x}^{\wedge} 9 *(2-\mathrm{r})^{\wedge} 5\right)-(1+\mathrm{h}) * \mathrm{~h}^{*}\left(\mathrm{x} * \mathrm{r}-(1 / 32)^{*} \mathrm{x}^{\wedge} 9 *(2-\mathrm{r})^{\wedge} 5\right)-\mathrm{h}^{*}\left(-\mathrm{h}^{\wedge} 5^{*}\left(-(1 / 45097156608) * \mathrm{r}^{\wedge} 25^{*} \mathrm{x}^{\wedge} 49+(31 / 5872025600 *(2-\right.\right.$ $\mathrm{r}))^{*} \mathrm{r}^{\wedge} 20 * \mathrm{x}^{\wedge} 41-(41 / 73400320) *(2-\mathrm{r})^{\wedge} 2^{*} \mathrm{r}^{\wedge} 15^{*} \mathrm{x}^{\wedge} 33+(3 / 81920) *(2-\mathrm{r})^{\wedge} 3 * \mathrm{r}^{\wedge} 10^{*} \mathrm{x}^{\wedge} 25-(3 / 2048)^{*}(2-\mathrm{r})^{\wedge} 4 * \mathrm{r}^{\wedge} 5 * \mathrm{x}^{\wedge} 17+(1 / 32)^{*} \mathrm{x}^{\wedge} 9^{*}(2-$ $\left.\left.\mathrm{r})^{\wedge} 5\right)\right)+(1+\mathrm{h})^{*}\left(-(1+\mathrm{h}) * \mathrm{~h} *\left(\mathrm{x}^{*} \mathrm{r}-(1 / 32)^{*} \mathrm{x}^{\wedge} 9^{*}(2-\mathrm{r})^{\wedge} 5\right)+\mathrm{h}^{\wedge} 6^{*}\left(-(1 / 45097156608) * \mathrm{r}^{\wedge} 25^{*} \mathrm{x}^{\wedge} 49+(31 / 5872025600 *(2-\mathrm{r}))^{*} \mathrm{r}^{\wedge} 20^{*} \mathrm{x}^{\wedge} 41-\right.\right.$ $\left.\left.(41 / 73400320)^{*}(2-r)^{\wedge} 2 * r^{\wedge} 15^{*} x^{\wedge} 33+(3 / 81920)^{*}(2-r)^{\wedge} 3 * r^{\wedge} 10 * x^{\wedge} 25-(3 / 2048)^{*}(2-r)^{\wedge} 4 * r^{\wedge} 5^{*} x^{\wedge} 17+(1 / 32)^{*} x^{\wedge} 9^{*}(2-r)^{\wedge} 5\right)\right)-$ $\mathrm{h} * \mathrm{x}^{*}\left(\left(1 / 40 *\left(-(1 / 4) *(1+\mathrm{h})^{\wedge} 3 * \mathrm{~h}^{\wedge} 3 *(2-\mathrm{r})^{\wedge} 3 *\left((41 / 36700160 *(1+\mathrm{h}))^{*} \mathrm{~h}^{\wedge} 7 *(2-\right.\right.\right.\right.$

$\mathrm{r})^{\wedge} 16^{*} \mathrm{r}^{\wedge} 2+\left(3 / 40960 *\left((1 / 32 *(1+\mathrm{h}))^{*} \mathrm{~h}^{*} \mathrm{r}^{\wedge} 5+(1 / 32)^{*} \mathrm{~h}^{\wedge} 6 * \mathrm{r}^{\wedge} 5\right)\right)^{*} \mathrm{~h}^{\wedge} 6 * \mathrm{r}^{\wedge} 3 *(2-\mathrm{r})^{\wedge} 10+(9 / 4194304) * \mathrm{~h}^{\wedge} 12 * \mathrm{r}^{\wedge} 8 *(2-$ $\left.\mathrm{r})^{\wedge} 10\right)+(1 / 4)^{*}(1+\mathrm{h})^{\wedge} 2^{*} \mathrm{~h}^{\wedge} 2 *(2-\mathrm{r})^{\wedge} 2^{*}\left((1 / 32 *(1+\mathrm{h}))^{*} \mathrm{~h}^{*} \mathrm{r}^{\wedge} 5+(1 / 32)^{*} \mathrm{~h}^{\wedge} 6^{*} \mathrm{r}^{\wedge} 5\right)^{*}\left(-(3 / 40960 *(1+\mathrm{h}))^{*} \mathrm{~h}^{\wedge} 7 *(2-\mathrm{r})^{\wedge} 11 * \mathrm{r}^{\wedge} 3-\right.$ $\left.\left(3 / 1024 *\left((1 / 32 *(1+\mathrm{h})) * h^{*} \mathrm{r}^{\wedge} 5+(1 / 32) * \mathrm{~h}^{\wedge} 6^{*} \mathrm{r}^{\wedge} 5\right)\right)^{*} \mathrm{~h}^{\wedge} 6 * \mathrm{r}^{\wedge} 4 *(2-\mathrm{r})^{\wedge} 5\right)+\left(-(1 / 20 *(1+\mathrm{h}))^{*} \mathrm{~h} *(2-\mathrm{r}) *\left((3 / 1024 *(1+\mathrm{h}))^{*} \mathrm{~h}^{\wedge} 7 *(2-\right.\right.$ $\left.\mathrm{r})^{\wedge} 6^{*} \mathrm{r}^{\wedge} 4+\left((1 / 32 *(1+\mathrm{h}))^{*} \mathrm{~h}^{*} \mathrm{r}^{\wedge} 5+(1 / 32)^{*} \mathrm{~h}^{\wedge} 6^{*} \mathrm{r}^{\wedge} 5\right)^{\wedge} 2\right)-(1 / 10)^{*}\left((1 / 32 *(1+\mathrm{h}))^{*} \mathrm{~h}^{*} \mathrm{r}^{\wedge} 5+(1 / 32)^{*} \mathrm{~h}^{\wedge} 6^{*} \mathrm{r}^{\wedge} 5\right)^{\wedge} 2^{*}(1+\mathrm{h}) * \mathrm{~h}^{*}(2-\mathrm{r})-$ $\left.(3 / 40960) * h^{\wedge} 8 * r^{\wedge} 4 *(2-r)^{\wedge} 7 *(1+h)^{\wedge} 2\right)^{*}\left((3 / 1024 *(1+h))^{*} h^{\wedge} 7 *(2-r)^{\wedge} 6 * r^{\wedge} 4+\left((1 / 32 *(1+h))^{*} h^{*} r^{\wedge} 5+(1 / 32)^{*} h^{\wedge} 6 * r^{*} 5\right)^{\wedge} 2\right)-(2 *(-$ $(1 / 28 *(1+\mathrm{h}))^{*} \mathrm{~h} *(2-\mathrm{r}) *\left(-(3 / 40960 *(1+\mathrm{h}))^{*} \mathrm{~h}^{\wedge} 7 *(2-\mathrm{r})^{\wedge} 11 * \mathrm{r}^{\wedge} 3-\left(3 / 1024 *\left((1 / 32 *(1+\mathrm{h}))^{*} \mathrm{~h}^{*} \mathrm{r}^{\wedge} 5+(1 / 32)^{*} \mathrm{~h}^{\wedge} 6 * \mathrm{r}^{\wedge} 5\right)\right)^{*} \mathrm{~h}^{\wedge} 6 * \mathrm{r}^{\wedge} 4 *(2-\right.$ $\left.\mathrm{r})^{\wedge} 5\right)+\left(1 / 28 *\left((1 / 32 *(1+\mathrm{h})) * \mathrm{~h}^{*} \mathrm{r}^{\wedge} 5+(1 / 32) * \mathrm{~h}^{\wedge} 6 * \mathrm{r}^{\wedge} 5\right)\right) *\left((3 / 1024 *(1+\mathrm{h}))^{*} \mathrm{~h}^{\wedge} 7 *(2-\right.$

$\left.\mathrm{r})^{\wedge} 6 * \mathrm{r}^{\wedge} 4+\left((1 / 32 *(1+\mathrm{h})) * \mathrm{~h}^{*} \mathrm{r}^{\wedge} 5+(1 / 32) * \mathrm{~h}^{\wedge} 6 * \mathrm{r}^{\wedge} 5\right)^{\wedge} 2\right)+(3 / 28672) * \mathrm{~h}^{\wedge} 7 * \mathrm{r}^{\wedge} 4 *(2-$

$\left.\left.\mathrm{r})^{\wedge} 6 *(1+\mathrm{h}) *\left((1 / 32 *(1+\mathrm{h})) * \mathrm{~h}^{*} \mathrm{r}^{\wedge} 5+(1 / 32)^{*} \mathrm{~h}^{\wedge} 6 * \mathrm{r}^{\wedge} 5\right)+(3 / 2293760) * \mathrm{~h}^{\wedge} 8 * \mathrm{r}^{\wedge} 3 *(2-\mathrm{r})^{\wedge} 12 *(1+\mathrm{h})^{\wedge} 2\right)\right)^{*}(1+\mathrm{h}) * \mathrm{~h}^{*}(2-$ $\mathrm{r})^{*}\left((1 / 32 *(1+\mathrm{h}))^{*} \mathrm{~h}^{*} \mathrm{r}^{\wedge} 5+(1 / 32)^{*} \mathrm{~h}^{\wedge} 6^{*} \mathrm{r}^{\wedge} 5\right)+\left(-(1 / 36 *(1+\mathrm{h}))^{*} \mathrm{~h} *(2-\mathrm{r}) *\left((41 / 36700160 *(1+\mathrm{h}))^{*} \mathrm{~h}^{\wedge} 7^{*}(2-\right.\right.$

$\mathrm{r})^{\wedge} 16 * \mathrm{r}^{\wedge} 2+\left(3 / 40960 *\left((1 / 32 *(1+\mathrm{h}))^{*} \mathrm{~h}^{*} \mathrm{r}^{\wedge} 5+(1 / 32)^{*} \mathrm{~h}^{\wedge} 6 * \mathrm{r}^{\wedge} 5\right)\right)^{*} \mathrm{~h}^{\wedge} 6 * \mathrm{r}^{\wedge} 3 *(2-\mathrm{r})^{\wedge} 10+(9 / 4194304)^{*} \mathrm{~h}^{\wedge} 12 * \mathrm{r}^{\wedge} 8 *(2-$ $\left.\mathrm{r})^{\wedge} 10\right)+\left(1 / 36 *\left((1 / 32 *(1+\mathrm{h})) * \mathrm{~h}^{*} \mathrm{r}^{\wedge} 5+(1 / 32) * \mathrm{~h}^{\wedge} 6^{*} \mathrm{r}^{\wedge} 5\right)\right) *\left(-(3 / 40960 *(1+\mathrm{h}))^{*} \mathrm{~h}^{\wedge} 7 *(2-\mathrm{r})^{\wedge} 11^{*} \mathrm{r}^{\wedge} 3-\right.$

$\left.\left(3 / 1024 *\left((1 / 32 *(1+h)) * h^{*} r^{\wedge} 5+(1 / 32) * h^{\wedge} 6 * r^{\wedge} 5\right)\right)^{*} h^{\wedge} 6 * r^{\wedge} 4 *(2-r) \wedge 5\right)-(1 / 24576) * h^{\wedge} 6 * r^{\wedge} 4 *(2-r)^{\wedge} 5 *\left((3 / 1024 *(1+h)) * h^{\wedge} 7 *(2-\right.$ $\left.\mathrm{r})^{\wedge} 6 * \mathrm{r}^{\wedge} 4+\left((1 / 32 *(1+\mathrm{h}))^{*} \mathrm{~h}^{*} \mathrm{r}^{\wedge} 5+(1 / 32)^{*} \mathrm{~h}^{\wedge} 6^{*} \mathrm{r}^{\wedge} 5\right)^{\wedge} 2\right)-(1 / 491520) * \mathrm{~h}^{\wedge} 7 * \mathrm{r}^{\wedge} 3 *(2-$

$\left.\mathrm{r})^{\wedge} 11^{*}(1+\mathrm{h}) *\left((1 / 32 *(1+\mathrm{h}))^{*} \mathrm{~h}^{*} \mathrm{r}^{\wedge} 5+(1 / 32) * \mathrm{~h}^{\wedge} 6 * \mathrm{r}^{\wedge} 5\right)-(41 / 2642411520)^{*} \mathrm{~h}^{\wedge} 8 * \mathrm{r}^{\wedge} 2 *(2-\mathrm{r})^{\wedge} 17 *(1+\mathrm{h})^{\wedge} 2\right)^{*}(1+\mathrm{h})^{\wedge} 2 * \mathrm{~h}^{\wedge} 2 *(2-$

$\left.\left.\mathrm{r})^{\wedge} 2\right)\right)^{*} \mathrm{x}^{\wedge} 40+\left(1 / 32 *\left(-(1 / 4)^{*}(1+\mathrm{h})^{\wedge} 3 * \mathrm{~h}^{\wedge} 3 *(2-\mathrm{r})^{\wedge} 3 *\left(-(3 / 40960 *(1+\mathrm{h}))^{*} \mathrm{~h}^{\wedge} 7 *(2-\mathrm{r})^{\wedge} 11^{*} \mathrm{r}^{\wedge} 3-\right.\right.\right.$

$\left.\left(3 / 1024 *\left((1 / 32 *(1+\mathrm{h})) * \mathrm{~h}^{*} \mathrm{r}^{\wedge} 5+(1 / 32) * \mathrm{~h}^{\wedge} 6 * \mathrm{r}^{\wedge} 5\right)\right)^{*} \mathrm{~h}^{\wedge} 6 * \mathrm{r}^{\wedge} 4 *(2-\mathrm{r})^{\wedge} 5\right)+(1 / 4) *(1+\mathrm{h})^{\wedge} 2 * \mathrm{~h}^{\wedge} 2 *(2-$

$\mathrm{r})^{\wedge} 2 *\left((1 / 32 *(1+\mathrm{h}))^{*} \mathrm{~h}^{*} \mathrm{r}^{\wedge} 5+(1 / 32)^{*} \mathrm{~h}^{\wedge} 6 * \mathrm{r}^{\wedge} 5\right)^{*}\left((3 / 1024 *(1+\mathrm{h}))^{*} \mathrm{~h}^{\wedge} 7 *(2-\mathrm{r})^{\wedge} 6 * \mathrm{r}^{\wedge} 4+\left((1 / 32 *(1+\mathrm{h}))^{*} \mathrm{~h}^{*} \mathrm{r}^{\wedge} 5+(1 / 32)^{*} \mathrm{~h}^{\wedge} 6 * \mathrm{r}^{\wedge} 5\right)^{\wedge} 2\right)-$ $\left(2 *\left(-(1 / 20 *(1+\mathrm{h})) * \mathrm{~h} *(2-\mathrm{r}) *\left((3 / 1024 *(1+\mathrm{h})) * \mathrm{~h}^{\wedge} 7 *(2-\mathrm{r})^{\wedge} 6 * \mathrm{r}^{\wedge} 4+\left((1 / 32 *(1+\mathrm{h})) * \mathrm{~h}^{*} \mathrm{r}^{\wedge} 5+(1 / 32)^{*} \mathrm{~h}^{\wedge} 6 * \mathrm{r}^{\wedge} 5\right)^{\wedge} 2\right)-\right.\right.$

$\left.\left.(1 / 10) *\left((1 / 32 *(1+h)) * h^{*} \mathrm{r}^{\wedge} 5+(1 / 32)^{*} \mathrm{~h}^{\wedge} 6^{*} \mathrm{r}^{\wedge} 5\right)^{\wedge} 2 *(1+\mathrm{h}) * \mathrm{~h}^{*}(2-\mathrm{r})-(3 / 40960) * \mathrm{~h}^{\wedge} 8 * \mathrm{r}^{\wedge} 4 *(2-\mathrm{r})^{\wedge} 7 *(1+\mathrm{h}){ }^{\wedge} 2\right)\right)^{*}(1+\mathrm{h}) * \mathrm{~h} *(2-$ $\mathrm{r}) *\left((1 / 32 *(1+\mathrm{h}))^{*} \mathrm{~h}^{*} \mathrm{r}^{\wedge} 5+(1 / 32) * \mathrm{~h}^{\wedge} 6 * \mathrm{r}^{\wedge} 5\right)+\left(-(1 / 28 *(1+\mathrm{h}))^{*} \mathrm{~h} *(2-\mathrm{r}) *\left(-(3 / 40960 *(1+\mathrm{h}))^{*} \mathrm{~h}^{\wedge} 7 *(2-\mathrm{r})^{\wedge} 11 * \mathrm{r}^{\wedge} 3-\right.\right.$ $\left(3 / 1024 *\left((1 / 32 *(1+h)) * h^{*} r^{\wedge} 5+(1 / 32) * h^{\wedge} 6 * r^{\wedge} 5\right.\right.$ 


$$
\begin{gathered}
\bar{u}_{0}(x, \alpha)=0 \\
\bar{u}_{1}(x, \alpha)=-\mathrm{h}^{*}\left(\mathrm{x}^{*}(2-\mathrm{r})-(1 / 32)^{*} \mathrm{x}^{\wedge} 9^{*} \mathrm{r}^{\wedge} 5\right)
\end{gathered}
$$

$\bar{u}_{2}(x, \alpha)=-(1+\mathrm{h}) * \mathrm{~h} *\left(\mathrm{x}^{*}(2-\mathrm{r})-(1 / 32)^{*} \mathrm{x}^{\wedge} 9^{*} \mathrm{r}^{\wedge} 5\right)-\mathrm{h} *\left(-\mathrm{h}^{\wedge} 5 *\left(-(1 / 45097156608) *(2-\mathrm{r})^{\wedge} 25^{*} \mathrm{x}^{\wedge} 49+(31 / 5872025600) * \mathrm{r}^{*}(2-\right.\right.$ $\left.\left.\mathrm{r})^{\wedge} 20^{*} \mathrm{x}^{\wedge} 41-(41 / 73400320)^{*} \mathrm{r}^{\wedge} 2 *(2-\mathrm{r})^{\wedge} 15^{*} \mathrm{x}^{\wedge} 33+(3 / 81920)^{*} \mathrm{r}^{\wedge} 3 *(2-\mathrm{r})^{\wedge} 10^{*} \mathrm{x}^{\wedge} 25-(3 / 2048)^{*} \mathrm{r}^{\wedge} 4 *(2-\mathrm{r})^{\wedge} 5^{*} \mathrm{x}^{\wedge} 17+(1 / 32)^{*} \mathrm{x}^{\wedge} 9^{*} \mathrm{r}^{\wedge} 5\right)\right)$

$\bar{u}_{3}(x, \alpha)=(1+\mathrm{h}) *\left(-(1+\mathrm{h}) * \mathrm{~h} *\left(\mathrm{x}^{*}(2-\mathrm{r})-(1 / 32) * \mathrm{x}^{\wedge} 9{ }^{*} \mathrm{r}^{\wedge} 5\right)+\mathrm{h}^{\wedge} 6^{*}\left(-(1 / 45097156608) *(2-\mathrm{r}) \wedge 25 * \mathrm{x}^{\wedge} 49+(31 / 5872025600) * \mathrm{r}^{*}(2-\right.\right.$ $\left.\left.\mathrm{r})^{\wedge} 20^{*} \mathrm{x}^{\wedge} 41-(41 / 73400320)^{*} \mathrm{r}^{\wedge} 2 *(2-\mathrm{r})^{\wedge} 15 * \mathrm{x}^{\wedge} 33+(3 / 81920)^{*} \mathrm{r}^{\wedge} 3 *(2-\mathrm{r})^{\wedge} 10^{*} \mathrm{x}^{\wedge} 25-(3 / 2048)^{*} \mathrm{r}^{\wedge} 4 *(2-\mathrm{r})^{\wedge} 5^{*} \mathrm{x}^{\wedge} 17+(1 / 32)^{*} \mathrm{x}^{\wedge} 9^{*} \mathrm{r}^{\wedge} 5\right)\right)-$ $\mathrm{h} * \mathrm{x} *\left(\left(1 / 136 *\left(\left(1 / 2033753534126478065664 *\left(-(1 / 36 *(1+\mathrm{h})) * \mathrm{~h} * \mathrm{r} *\left((41 / 36700160) * \mathrm{~h}^{\wedge} 7 *(2-\right.\right.\right.\right.\right.\right.$

$\mathrm{r})^{\wedge} 2 * \mathrm{r}^{\wedge} 16^{*}(1+\mathrm{h})+\left(3 / 40960 *\left((1 / 32 *(1+\mathrm{h}))^{*} \mathrm{~h} *(2-\mathrm{r})^{\wedge} 5+(1 / 32)^{*} \mathrm{~h}^{\wedge} 6 *(2-\mathrm{r})^{\wedge} 5\right)\right)^{*} \mathrm{~h}^{\wedge} 6 *(2-\mathrm{r})^{\wedge} 3^{*} \mathrm{r}^{\wedge} 10+(9 / 4194304)^{*} \mathrm{~h}^{\wedge} 12 *(2-$ $\left.\mathrm{r})^{\wedge} 8^{*} \mathrm{r}^{\wedge} 10\right)+\left(1 / 36^{*}\left((1 / 32 *(1+\mathrm{h}))^{*} \mathrm{~h} *(2-\mathrm{r})^{\wedge} 5+(1 / 32)^{*} \mathrm{~h}^{\wedge} 6 *(2-\mathrm{r})^{\wedge} 5\right)\right)^{*}\left(-(3 / 40960)^{*} \mathrm{~h}^{\wedge} 7 *(2-\mathrm{r})^{\wedge} 3 * \mathrm{r}^{\wedge} 11 *(1+\mathrm{h})-\right.$ $\left.\left(3 / 1024 *\left((1 / 32 *(1+\mathrm{h}))^{*} \mathrm{~h} *(2-\mathrm{r})^{\wedge} 5+(1 / 32)^{*} \mathrm{~h}^{\wedge} 6 *(2-\mathrm{r})^{\wedge} 5\right)\right)^{*} \mathrm{~h}^{\wedge} 6^{*}(2-\mathrm{r})^{\wedge} 4 * \mathrm{r}^{\wedge} 5\right)-(1 / 24576) * \mathrm{~h}^{\wedge} 6^{*}(2-\mathrm{r})^{\wedge} 4 * \mathrm{r}^{\wedge} 5^{*}\left((3 / 1024)^{*} \mathrm{~h}^{\wedge} 7^{*}(2-\right.$ $\left.\mathrm{r})^{\wedge} 4 * \mathrm{r}^{\wedge} 6 *(1+\mathrm{h})+\left((1 / 32 *(1+\mathrm{h}))^{*} \mathrm{~h} *(2-\mathrm{r})^{\wedge} 5+(1 / 32)^{*} \mathrm{~h}^{\wedge} 6 *(2-\mathrm{r})^{\wedge} 5\right)^{\wedge} 2\right)-(1 / 491520)^{*} \mathrm{~h}^{\wedge} 7^{*}(2-\mathrm{r})^{\wedge} 3 *{ }^{\wedge} \mathrm{r}^{\wedge} 11^{*}(1+\mathrm{h}) *\left((1 / 32 *(1+\mathrm{h}))^{*} \mathrm{~h}^{*}(2-\right.$ r)^5+(1/32)* $\left.\left.\left.\mathrm{h}^{\wedge} 6 *(2-\mathrm{r})^{\wedge} 5\right)-(41 / 2642411520)^{*} \mathrm{~h}^{\wedge} 8^{*}(2-\mathrm{r})^{\wedge} 2 * \mathrm{r}^{\wedge} 17 *(1+\mathrm{h})^{\wedge} 2\right)\right)^{*} \mathrm{~h}^{\wedge} 12 * \mathrm{r}^{\wedge} 50-(31 / 132405829044692582400 *(-$ $(1 / 44 *(1+\mathrm{h})) * \mathrm{~h}^{*} \mathrm{r} *\left(-(31 / 2936012800) * \mathrm{~h}^{\wedge}{ }^{\wedge} *(2-\mathrm{r})^{*} \mathrm{r}^{\wedge} 21 *(1+\mathrm{h})-\left(41 / 36700160 *\left((1 / 32 *(1+\mathrm{h}))^{*} \mathrm{~h} *(2-\mathrm{r})^{\wedge} 5+(1 / 32) * \mathrm{~h}^{\wedge} 6 *(2-\right.\right.\right.$ $\left.\left.\left.\mathrm{r})^{\wedge} 5\right)\right)^{*} \mathrm{~h}^{\wedge} 6 *(2-\mathrm{r})^{\wedge} 2 * \mathrm{r}^{\wedge} 15-(9 / 83886080)^{*} \mathrm{~h}^{\wedge} 12 *(2-\mathrm{r})^{\wedge} 7 * \mathrm{r}^{\wedge} 15\right)+\left(1 / 44 *\left((1 / 32 *(1+\mathrm{h}))^{*} \mathrm{~h} *(2-\mathrm{r})^{\wedge} 5+(1 / 32)^{*} \mathrm{~h}^{\wedge} 6 *(2-\right.\right.$ r) $\left.\left.\wedge^{\wedge}\right)\right)^{*}\left((41 / 36700160)^{*} \mathrm{~h}^{\wedge} 7 *(2-\mathrm{r})^{\wedge} 2 * \mathrm{r}^{\wedge} 16^{*}(1+\mathrm{h})+\left(3 / 40960 *\left((1 / 32 *(1+\mathrm{h}))^{*} \mathrm{~h} *(2-\mathrm{r})^{\wedge} 5+(1 / 32)^{*} \mathrm{~h}^{\wedge} 6 *(2-\mathrm{r})^{\wedge} 5\right)\right)^{*} \mathrm{~h}^{\wedge} 6^{*}(2-\right.$ $\mathrm{r})^{\wedge} 3 * \mathrm{r}^{\wedge} 10+(9 / 4194304) * \mathrm{~h}^{\wedge} 12 *(2-\mathrm{r})^{\wedge} 8^{*} \mathrm{r}^{\wedge} 10$

$$
\bar{u}(x, \alpha)=\bar{u}_{0}(x, \alpha)+\sum_{m=1}^{2} \bar{u}_{m}(x, \alpha)
$$

$\bar{u}(x, \alpha)=$

$\mathrm{h} *\left(\mathrm{x}^{*}(2-\mathrm{r})-(1 / 32) * \mathrm{x}^{\wedge} 9 * \mathrm{r} \mathrm{r}^{\wedge}\right)-(1+\mathrm{h}) * \mathrm{~h} *\left(\mathrm{x} *(2-\mathrm{r})-(1 / 32) * \mathrm{x}^{\wedge} 9 * \mathrm{r}^{\wedge} 5\right)-\mathrm{h} *\left(-\mathrm{h}^{\wedge} 5 *(-(1 / 45097156608) *(2-\right.$

r)^25* $\mathrm{x}^{\wedge} 49+(31 / 5872025600) * \mathrm{r}^{*}(2-\mathrm{r})^{\wedge} 20^{*} \mathrm{x}^{\wedge} 41-(41 / 73400320) * \mathrm{r}^{\wedge} 2 *(2-\mathrm{r})^{\wedge} 15^{*} \mathrm{x}^{\wedge} 33+(3 / 81920) * \mathrm{r}^{\wedge} 3 *(2-\mathrm{r})^{\wedge} 10^{*} \mathrm{x}^{\wedge} 25-$

$\left.\left.(3 / 2048) * \mathrm{r}^{\wedge} 4 *(2-\mathrm{r})^{\wedge} 5 * \mathrm{x}^{\wedge} 17+(1 / 32) * \mathrm{x}^{\wedge} 9 * \mathrm{r}^{\wedge} 5\right)\right)+(1+\mathrm{h}) *\left(-(1+\mathrm{h}) * \mathrm{~h} *\left(\mathrm{x}^{*}(2-\mathrm{r})-(1 / 32) * \mathrm{x}^{\wedge}{ }^{*}{ }^{*} \mathrm{r}^{\wedge} 5\right)+\mathrm{h}^{\wedge} 6 *(-(1 / 45097156608) *(2-\right.$ r)^25* $\mathrm{x}^{\wedge} 49+(31 / 5872025600) * \mathrm{r}^{*}(2-\mathrm{r})^{\wedge} 20^{*} \mathrm{x}^{\wedge} 41-(41 / 73400320) * \mathrm{r}^{\wedge} 2 *(2-\mathrm{r})^{\wedge} 15^{*} \mathrm{x}^{\wedge} 33+(3 / 81920) * \mathrm{r}^{\wedge} 3 *(2-\mathrm{r})^{\wedge} 10^{*} \mathrm{x}^{\wedge} 25-$ $\left.\left.(3 / 2048) * \mathrm{r}^{\wedge} 4 *(2-\mathrm{r})^{\wedge} 5^{*} \mathrm{x}^{\wedge} 17+(1 / 32) * \mathrm{x}^{\wedge} 9^{*} \mathrm{r}^{\wedge} 5\right)\right)-\mathrm{h} * \mathrm{x}^{*}((1 / 136 *((1 / 2033753534126478065664 *(-$

$(1 / 36 *(1+\mathrm{h})) * \mathrm{~h}^{*} \mathrm{r}^{*}\left((41 / 36700160) * \mathrm{~h}^{\wedge} 7 *(2-\mathrm{r}){ }^{\wedge} 2 * \mathrm{r}^{\wedge} 16 *(1+\mathrm{h})+\left(3 / 40960 *\left((1 / 32 *(1+\mathrm{h}))^{*} \mathrm{~h} *(2-\mathrm{r})^{\wedge} 5+(1 / 32) * \mathrm{~h}^{\wedge} 6 *(2-\right.\right.\right.$

r $\left.\left.\left.)^{\wedge} 5\right)\right)^{*} \mathrm{~h}^{\wedge} 6 *(2-\mathrm{r})^{\wedge} 3 * \mathrm{r}^{\wedge} 10+(9 / 4194304)^{*} \mathrm{~h}^{\wedge} 12 *(2-\mathrm{r})^{\wedge} 8^{*} \mathrm{r}^{\wedge} 10\right)+\left(1 / 36 *\left((1 / 32 *(1+\mathrm{h}))^{*} \mathrm{~h}^{*}(2-\mathrm{r})^{\wedge} 5+(1 / 32) * \mathrm{~h}^{\wedge} 6^{*}(2-\mathrm{r})^{\wedge} 5\right)\right)^{*}(-$ $\left.(3 / 40960)^{*} \mathrm{~h}^{\wedge} 7 *(2-\mathrm{r})^{\wedge} 3 * \mathrm{r}^{\wedge} 11 *(1+\mathrm{h})-\left(3 / 1024 *\left((1 / 32 *(1+\mathrm{h}))^{*} \mathrm{~h} *(2-\mathrm{r})^{\wedge} 5+(1 / 32) * \mathrm{~h}^{\wedge} 6 *(2-\mathrm{r})^{\wedge} 5\right)\right)^{*} \mathrm{~h}^{\wedge} 6 *(2-\mathrm{r})^{\wedge} 4 * \mathrm{r}^{\wedge} 5\right)-$ $(1 / 24576) * \mathrm{~h}^{\wedge} 6 *(2-\mathrm{r})^{\wedge} 4 * \mathrm{r}^{\wedge} 5 *\left((3 / 1024) * \mathrm{~h}^{\wedge} 7 *(2-\mathrm{r})^{\wedge} 44^{*} \mathrm{r}^{\wedge} 6 *(1+\mathrm{h})+\left((1 / 32 *(1+\mathrm{h}))^{*} \mathrm{~h}^{*}(2-\mathrm{r})^{\wedge} 5+(1 / 32)^{*} \mathrm{~h}^{\wedge} 6 *(2-\mathrm{r})^{\wedge} 5\right)^{\wedge} 2\right)-$ $(1 / 491520)^{*} \mathrm{~h}^{\wedge} 7 *(2-\mathrm{r})^{\wedge} 3 * \mathrm{r}^{\wedge} 11^{*}(1+\mathrm{h}) *\left((1 / 32 *(1+\mathrm{h}))^{*} \mathrm{~h}^{*}(2-\mathrm{r})^{\wedge} 5+(1 / 32)^{*} \mathrm{~h}^{\wedge} 6^{*}(2-\mathrm{r})^{\wedge} 5\right)-(41 / 2642411520)^{*} \mathrm{~h}^{\wedge} 8^{*}(2-$ $\left.\left.\mathrm{r})^{\wedge} 2 * \mathrm{r}^{\wedge} 17 *(1+\mathrm{h})^{\wedge} 2\right)\right) * \mathrm{~h}^{\wedge} 12 * \mathrm{r}^{\wedge} 50-\left(31 / 132405829044692582400 *\left(-(1 / 44 *(1+\mathrm{h})) * \mathrm{~h}^{*} \mathrm{r}^{*}\left(-(31 / 2936012800) * \mathrm{~h}^{\wedge} 7 *(2-\right.\right.\right.$ r) $)^{*} \mathrm{r}^{\wedge} 21 *(1+\mathrm{h})-\left(41 / 36700160 *\left((1 / 32 *(1+\mathrm{h})) * \mathrm{~h} *(2-\mathrm{r})^{\wedge} 5+(1 / 32)^{*} \mathrm{~h}^{\wedge} 6 *(2-\mathrm{r})^{\wedge} 5\right)\right)^{*} \mathrm{~h}^{\wedge} 6^{*}(2-\mathrm{r})^{\wedge} 2 * \mathrm{r}^{\wedge} 15-(9 / 83886080) * \mathrm{~h}^{\wedge} 12 *(2-$ r) $\left.\wedge^{\wedge}{ }^{*} \mathrm{r}^{\wedge} 15\right)+\left(1 / 44 *\left((1 / 32 *(1+\mathrm{h}))^{*} \mathrm{~h} \mathrm{~h}^{*}(2-\mathrm{r})^{\wedge} 5+(1 / 32)^{*} \mathrm{~h}^{\wedge} 6 *(2-\mathrm{r})^{\wedge} 5\right)\right)^{*}\left((41 / 36700160)^{*} \mathrm{~h}^{\wedge} 7^{\wedge}(2-\right.$

$\mathrm{r})^{\wedge} 2^{*} \mathrm{r}^{\wedge} 16^{*}(1+\mathrm{h})+\left(3 / 40960 *\left((1 / 32 *(1+\mathrm{h}))^{*} \mathrm{~h} \mathrm{~h}^{*}(2-\mathrm{r})^{\wedge} 5+(1 / 32)^{*} \mathrm{~h}^{\wedge} 6 *(2-\mathrm{r})^{\wedge} 5\right)\right)^{*} \mathrm{~h}^{\wedge} 6^{*}(2-\mathrm{r})^{\wedge} 3^{*} \mathrm{r}^{\wedge} 10+(9 / 4194304) * \mathrm{~h}^{\wedge} 12 *(2-$ r) $\left.\wedge^{*} \mathrm{r}^{\wedge} 10\right)-(3 / 90112)^{*} \mathrm{~h}^{\wedge} 6 *(2-\mathrm{r})^{\wedge} 4 * \mathrm{r}^{\wedge} 5^{*}\left(-(3 / 40960) * \mathrm{~h}^{\wedge} 7^{*}(2-\mathrm{r})^{\wedge} 3 * \mathrm{r}^{\wedge} 11 *(1+\mathrm{h})-\left(3 / 1024 *\left((1 / 32 *(1+\mathrm{h}))^{*} \mathrm{~h} *(2-\right.\right.\right.$ r) $\left.\left.\left.\left.)^{\wedge}+(1 / 32)^{*} \mathrm{~h}^{\wedge} 6 *(2-\mathrm{r})^{\wedge} 5\right)\right)^{*} \mathrm{~h}^{\wedge}\right)^{*}(2-\mathrm{r})^{\wedge} 4 * \mathrm{r}^{\wedge} 5\right)+(3 / 3604480)^{*} \mathrm{~h}^{\wedge} 6 *(2-\mathrm{r})^{\wedge} 3 * \mathrm{r}^{\wedge} 10 *\left((3 / 1024)^{*} \mathrm{~h}^{\wedge} 7 *(2-\right.$ r) $\left.\wedge^{\wedge} *^{*} \mathrm{r}^{\wedge} 6 *(1+\mathrm{h})+\left((1 / 32 *(1+\mathrm{h}))^{*} \mathrm{~h}^{*}(2-\mathrm{r})^{\wedge} 5+(1 / 32)^{*} \mathrm{~h}^{\wedge} 6^{*}(2-\mathrm{r})^{\wedge} 5\right)^{\wedge} 2\right)+(41 / 1614807040)^{*} \mathrm{~h}^{\wedge} 7^{*}(2-$ r) $\left.\left.2^{*} \mathrm{r}^{\wedge} 16^{*}(1+\mathrm{h}) *\left((1 / 32 *(1+\mathrm{h}))^{*} \mathrm{~h}^{*}(2-\mathrm{r})^{\wedge} 5+(1 / 32)^{*} \mathrm{~h}^{\wedge} 6^{*}(2-\mathrm{r})^{\wedge} 5\right)+(31 / 258369126400)^{*} \mathrm{~h}^{\wedge} 8^{*}(2-\mathrm{r})^{*} \mathrm{r}^{\wedge} 22^{*}(1+\mathrm{h})^{\wedge} 2\right)\right)^{*} \mathrm{~h}^{\wedge} 12^{*}(2-$ r) $* \mathrm{r}^{\wedge} 45+(10891 / 206884107882332160000 *(-$

$(1 / 52 *(1+\mathrm{h})) * \mathrm{~h}^{*} \mathrm{r} *\left((1 / 22548578304) * \mathrm{~h}^{\wedge} 7 * \mathrm{r}^{\wedge} 26 *(1+\mathrm{h})+\left(31 / 2936012800 *\left((1 / 32 *(1+\mathrm{h})) * \mathrm{~h} *(2-\mathrm{r}){ }^{\wedge} 5+(1 / 32) * \mathrm{~h}^{\wedge} 6 *(2-\right.\right.\right.$ r)^5) $)^{*} \mathrm{~h}^{\wedge} 6^{*}(2-\mathrm{r})^{*} \mathrm{r}^{\wedge} 20+(1119 / 375809638400) * \mathrm{~h}^{\wedge} 12$

Table 2. Computation between the exact and HAM $h=-1$ in case 2 and determine the absolute error.

\begin{tabular}{lllllll}
\hline $\boldsymbol{x}$ & $\boldsymbol{e x a c t} \underline{\boldsymbol{u}}(\boldsymbol{x}, \boldsymbol{r})$ & $\boldsymbol{H A M} \underline{\boldsymbol{u}}(\boldsymbol{x}, \boldsymbol{r}) \boldsymbol{\alpha}=\mathbf{0 . 1}$ & $\boldsymbol{e x a c t} \underline{u}(\boldsymbol{x}, \boldsymbol{r})$ & $\boldsymbol{H A M} \underline{\boldsymbol{u}}(\boldsymbol{x}, \boldsymbol{r}) \boldsymbol{\alpha}=\mathbf{0 . 5}$ & $\boldsymbol{e x a c t} \underline{\boldsymbol{u}}(\boldsymbol{x}, \boldsymbol{r})$ & $\boldsymbol{H A M} \underline{u}(\boldsymbol{x}, \boldsymbol{r}) \boldsymbol{\alpha}=\mathbf{1}$ \\
\hline 0 & 0.0000000 & 0.00000 & 0.000000 & 0.00000 & 0.00000 & 0.00000 \\
0.2 & 0.0200000 & 0.02000 & 0.100000 & 0.10000 & 0.20000 & 0.20000 \\
0.4 & 0.0400000 & 0.04000 & 0.200000 & 0.20000 & 0.40000 & 0.39999 \\
0.6 & 0.0600000 & 0.05999 & 0.300000 & 0.29999 & 0.60000 & 0.59999 \\
0.8 & 0.0800000 & 0.07999 & 0.400000 & 0.39999 & 0.80000 & 0.79999 \\
1 & 0.100000 & 0.09999 & 0.500000 & 0.49999 & 1.00000 & 0.99999 \\
\hline
\end{tabular}




\begin{tabular}{|c|c|c|c|c|c|c|}
\hline$x$ & exact $\bar{u}(x, r)$ & $H A M \bar{u}(x, r) \alpha=0.1$ & $\operatorname{exact} \bar{u}(x, r)$ & $H A M \bar{u}(x, r) \alpha=0.5$ & $\operatorname{exact} \bar{u}(x, r)$ & $H A M \bar{u}(x, r) \alpha=1$ \\
\hline 0 & 0.00000 & 0.00000 & 0.00000 & 0.00000 & 0.00000 & 0.00000 \\
\hline 0.2 & 0.38000 & 0.38000 & 0.30000 & 0.30000 & 0.20000 & 0.20000 \\
\hline 0.4 & 0.76000 & 0.76999 & 0.60000 & 0.59999 & 0.40000 & 0.39999 \\
\hline 0.6 & 1.14000 & 1.14000 & 0.90000 & 0.89998 & 0.60000 & 0.59999 \\
\hline 0.8 & 1.52000 & 1.51996 & 1.20000 & 1.19998 & 0.80000 & 0.79999 \\
\hline 1 & 1.90000 & 1.8999 & 1.50000 & 1.49952 & 1.00000 & 0.99999 \\
\hline
\end{tabular}

As long as a series solution given by the HAM converges, it must be the exact solution. So it is important to ensure that the homotopy solution series converges. contains the auxiliary parameter $\hbar$, which provides us a simple way to adjust and control the convergence of the solution series. In general, by means of the so-called $\hbar$-curve (a curve of a versus $\hbar)$, it is straightforward to choose an appropriate range for $\hbar$ which ensures the convergence of the solution series. As pointed by Liao [13], the valid region of $\hbar$ is a horizontal line segment. In Fig. (3a), Fig (2b) and Fig (4a), Fig (4b) we plot the $\hbar$-curves, we could find that if $\hbar$ is about in area $[-1,-0.7]$ the result is different values of $\hbar$

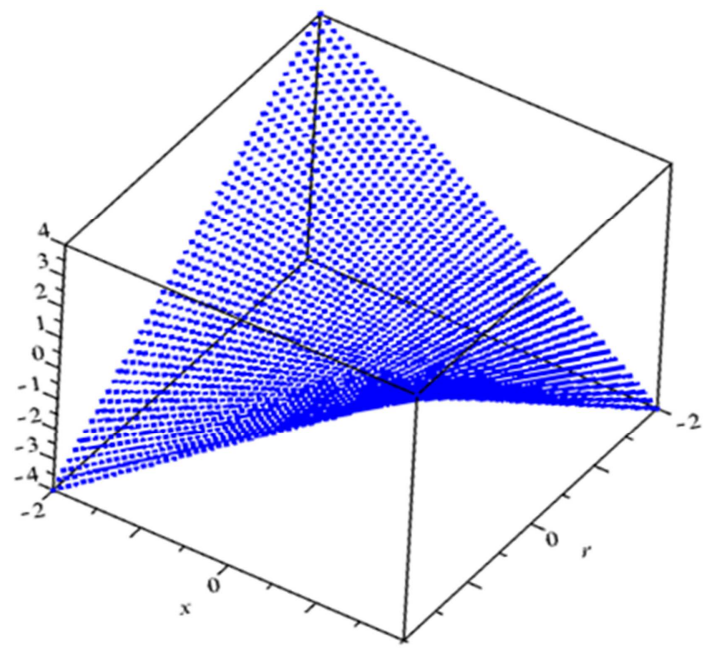

Figure 3a. The exact solution $\underline{\boldsymbol{u}}(\boldsymbol{x}, \boldsymbol{\alpha})$.

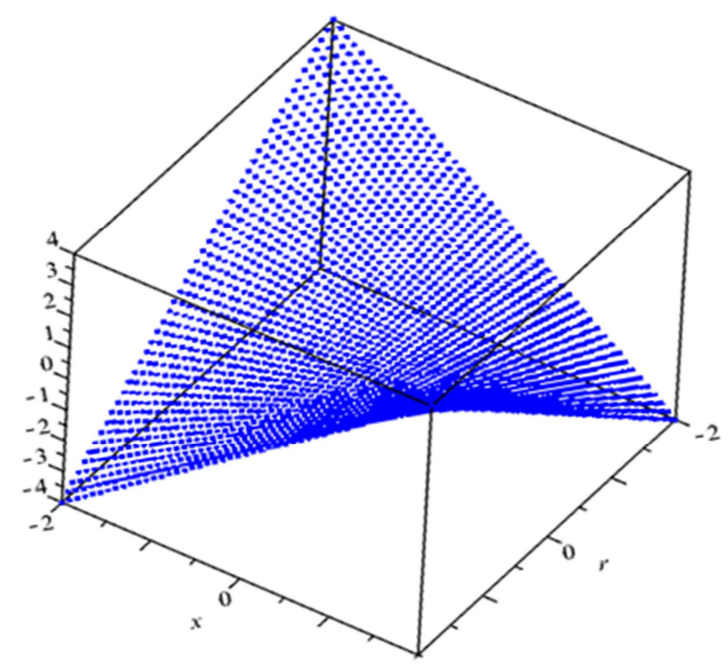

Figure $3 \boldsymbol{b}$. The approximte solution $\underline{\boldsymbol{u}}(\boldsymbol{x}, \boldsymbol{\alpha})$.

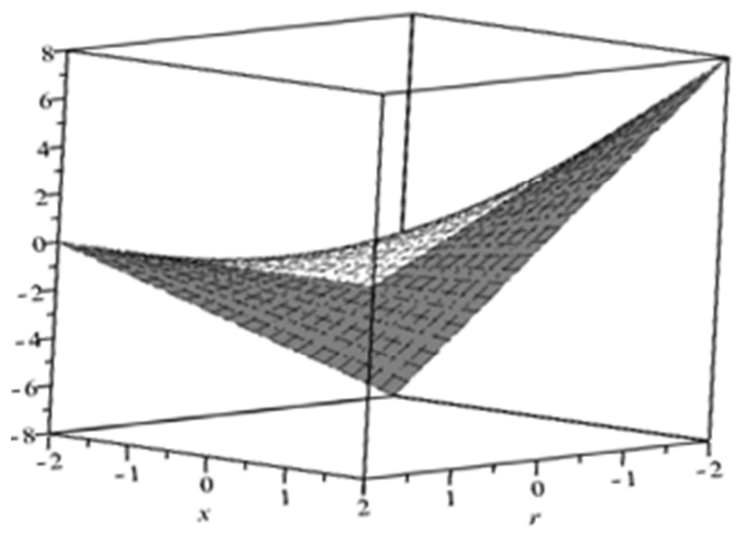

Figure 4a. The exact solution $\overline{\boldsymbol{u}}(\boldsymbol{x}, \boldsymbol{\alpha})$.

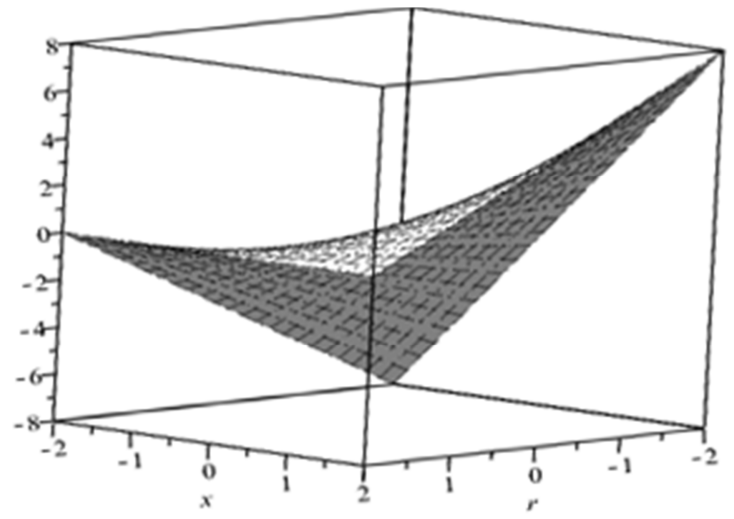

Figure $4 \boldsymbol{b}$. The approoximte solution $\overline{\boldsymbol{u}}(\boldsymbol{x}, \boldsymbol{\alpha})$.

\section{Conclusion}

The proposed method is a powerful procedure for solving fuzzy nonlinear Volterra integral equation. The examples analyzed illustrate the ability and reliability of the method presented in this paper and severals that the one is very simple and effective. The obtained solutions, in comparision with the exact solution admit a remarkable accuracy. Resultes indicate that the convergence rate is very fast, and lower approximations can achieve high accuracy

\section{References}

[1] Nor hanim abd. rahman, arsmah Ibrahim, mohd idris jays, newton homotopy solution for nonlinear equations using maple 14, faculty of computer and mathematical scienes university teknologi mara malyusia (uitm) 40450 Shah Alam, Selangor, Malaysia volume. 3, number 2 Decamber (2011). 
[2] M. Ghanbari, numerical solution of fuzzy linear volterra integral equation of the second kind by homotopy analysis method, Department of mathematics, science and research branch, Islamic Azad University, Tehran, Iran, Received 10 january 2010.

[3] Eman A. Hussain, Ayad W. Ali, Homotopy Analysis Method for solving Non linear fuzzy integral equation, Department of Mathematics, College of Science, Al-Mustansiriyah Baghdad, Iraq (2011).

[4] Edyta H. Damian Slota, Tomasz. T, Roman Wituda. Usage of the Homotopy analysis method for solving the Non Linear and linear integral equation of the second kind (2013). Northeland.

[5] R. Goetschel and Vaxman, Elementry fuzzy clculus, Fuzzy Sets and Systems, 18 (1986), 31-43.

[6] Sarmad A. Altari, Numerical Solutions of Fuzzy Fredholm Integral Equation of the second kind using Bernstein Polynomials, Department of Computer Engineering and Information Technology University of Technology, Baghdad Iraq (2012).

[7] Hany. N. Magdy. A, A new technique of using Homotopy Analysis Method for Second Order Non Linear Differential Equation, Department of Basic Science, Faculty of Engineering of Branch Benha University, Egypt (2012).

[8] N. A. Rajab, A. M. Ahmad, O. M. Alfaour, Reduction Formula for Linear Fuzzy Equation, Applied Science Department, University of Technology Baghdad - Iraq (2013).

[9] Sushila Rathora, Devendra Kumar, Jagdev Singh, Sumit Gapta, Homotopy Analysis Method for Non Linear Equation, Department of Mathematics, Jagon Meth, University Village Rampun Tehsil Chaksu, Jaipur - 303901, Ragashtan - India (2012).

[10] S. Abbasbandy, E. Babolian, M. Alavi, Numerical method for solving linear Fredholm fuzzy integral equations of the second kind, Chaos Soliton and Fractals 31 (2007) 138146.

[11] Abbasbandy, S., Msgyai, E., \& Shivanian, E. (2009). The homotopy analysis method for multiple solutions of nonlinear boundary value problems. Commun Nonlinear Sci. Num. Simulat., 14(9-10), 3530-3536.

[12] H.C. Wu, The improper fuzzy Riemann integral and its numerical integration, Information Science 111 (1999) 109137.
[13] S. Abbasbandy and A. Jafarian, "Steepest descent method for solving fuzzy nonlinear equations," Applied Mathematics and Computation, vol. 174, no. 1, pp. 669-675, 2006.

[14] .H. C. Wu, The proper fuzzy Riemann integral and its numerical integration, Information Science 111(1999) 109137.

[15] G. J. Klir, U. St. Clair, and B. Yuan, Fuzzy Set Theory: Foundations and Applications, Prentice-Hall, Eaglewood Cliffs, NJ, USA, 1997.

[16] J. Y. Park, Y. C. Kwan, J. V. Jeong, Existence of solutions of fuzzy integral equationsin Banach spaces, Fuzzy Sets and System 72 (1995) 373-378.

[17] Chang, S. S. L., \& Zadah, L. A. (1972). On fuzzy mapping and control. Trans. Systems, Man Cyberetics, SMC-2(1), 3034.

[18] S. Abbasbandy, "The application of homotopy nanlysis mthod to nonlinear equations arising in heat transfer," Phys. Lett. A, vol.360, pp 109-113, 2006.

[19] S. J. Liao, Notes on the homotopy analysis method: Some definitions and theorems, Commun Nonlinear Sci. Numer. Simulat. 14 (2009) 983-997.

[20] J. Y. Park, Y. C. Kwan, J. V. Jeong, Existence of solution of fuzzy integral equations in Banach spaces, Fuzzy Sets and System 72 ( 1995) 373- 378.

[21] D. Dubois, H. Prade, Operation on fuzzy numbers, Int. J. $\begin{array}{llll}\text { system } & \text { Science } & 9 & \text { (1978) }\end{array}$ http://dx.doi.org/10.1080/00207727808941724

[22] Nanda, S. (1989). On integration of fuzzy mappings. Fuzzy Sets and Systems, 32, 95-101.

[23] R. Goetschel and W. Vaxman, Elementary fuzzy calculus, Fuzzy Sets and Systems, 18 (1986), 31-43

[24] M. Mizumoto, K. Tanaka, The four operations of arithmetic on fuzzy numbers, Systems Comput. Controls 7(1976) 73-81.

[25] O. Kaleva, Fuzzy differential equations, Fuzzy Sets and Systems 24 (1987) 301-317 http://dx.doi.org/10.1016/01650114(87)90029-7. 